\title{
Derived categories of irreducible projective curves of arithmetic genus one
}

\author{
Igor Burban and Bernd Kreußler \\ Dedicated to Yuriy Drozd on his 60th birthday
}

\begin{abstract}
We investigate the bounded derived category of coherent sheaves on irreducible singular projective curves of arithmetic genus one. A description of the group of exact autoequivalences and the set of all $t$-structures of this category is given. We describe the moduli space of stability conditions, obtain a complete classification of all spherical objects in this category and show that the group of exact auto-equivalences acts transitively on them. Harder-Narasimhan filtrations in the sense of Bridgeland are used as our main technical tool.
\end{abstract}

\section{Introduction}

The purpose of this paper is to study the structure of the bounded derived category $D_{\text {coh }}^{b}(\boldsymbol{E})$ of coherent sheaves on a singular irreducible projective curve $\boldsymbol{E}$ of arithmetic genus one.

In the smooth case, such structure results are easily obtained from Atiyah's description [Ati57] of indecomposable vector bundles over elliptic curves. However, if $\boldsymbol{E}$ has a node or a cusp, some crucial properties fail to hold. This is illustrated by Table 1. Despite these difficulties, the main goal of this paper is to find the common features between the smooth and the singular case. A list of such can be found in Remark 5.13.

In $\S 2$, we review the smooth case and highlight where the properties mentioned above are used. Our approach was inspired by [LM93]. Atiyah's algorithm to construct indecomposable vector bundles of any slope can be understood as an application of a sequence of twist functors with spherical objects. From this point of view, Atiyah shows that any indecomposable object of $D_{\text {coh }}^{\mathrm{b}}(\boldsymbol{E})$ is the image of an indecomposable torsion sheaf under an exact auto-equivalence of $\mathrm{D}_{\text {coh }}^{\mathrm{b}}(\boldsymbol{E})$.

In the case of a singular Weierstraß curve $\boldsymbol{E}$, as our main technical tool we use HarderNarasimhan filtrations in $\mathrm{D}_{\mathrm{coh}}^{\mathrm{b}}(\boldsymbol{E})$, which were introduced by Bridgeland [Bri02]. Their general properties are studied in $\S 3$.

The key result of $\S 4$ is the preservation of stability under Seidel-Thomas twists [ST01] with spherical objects. This allows us to show that, like in the smooth case, any category of semi-stable sheaves with fixed slope is equivalent to the category of coherent torsion sheaves on $\boldsymbol{E}$.

In the case of slope zero, this was shown in our previous work [BK05]. For the nodal case, an explicit description of semi-stable sheaves of degree zero via étale coverings was given there as well. A combinatorial description of semi-stable sheaves of arbitrary slope over a nodal cubic curve was found by Mozgovoy [Moz04]. On the other hand, a classification of all indecomposable objects

Received 28 June 2005, accepted in final form 26 January 2006.

2000 Mathematics Subject Classification 18E30, 14H60, 14H52, 14H20, $14 \mathrm{H} 10$.

Keywords: derived category, elliptic curve, Fourier-Mukai transform, Harder-Narasimhan filtration, stability.

This journal is (c) Foundation Compositio Mathematica 2006. 


\section{Burban And B. Kreußler}

TABLE 1. Difficulties on singular curves.

\begin{tabular}{|c|c|c|}
\hline & Smooth & Singular \\
\hline homological dimension of $\mathrm{Coh}_{E}$ & 1 & $\infty$ \\
\hline Serre duality holds & in general & $\begin{array}{l}\text { with one object } \\
\text { being perfect }\end{array}$ \\
\hline torsion free implies locally free & yes & no \\
\hline $\begin{array}{l}\text { indecomposable coherent sheaves } \\
\text { are semi-stable }\end{array}$ & yes & no \\
\hline $\begin{array}{l}\text { any indecomposable complex is } \\
\text { isomorphic to a shift of a sheaf }\end{array}$ & yes & no \\
\hline
\end{tabular}

of $\mathrm{D}_{\text {coh }}^{\mathrm{b}}(\boldsymbol{E})$ was presented in [BD04b]. A description of the Harder-Narasimhan filtrations in terms of this classification is a task for future work.

However, if the singular point of $\boldsymbol{E}$ is a cusp, the description of all indecomposable coherent torsion sheaves is a wild problem in the sense of representation theory, see for example [Dro72]. Nevertheless, stable vector bundles on a cuspidal cubic have been classified by Bodnarchuk and Drozd [BD03].

It turns out that semi-stable sheaves of infinite homological dimension are particularly important, because only such sheaves appear as Harder-Narasimhan factors of indecomposable objects in $\mathrm{D}_{\mathrm{coh}}^{\mathrm{b}}(\boldsymbol{E})$ which are not semi-stable (Proposition 4.6).

The main result (Proposition 4.13) of $\S 4$ is the answer to a question of Polishchuk [Pol02, $\S 1.4$ ], who asked for a description of all spherical objects on $\boldsymbol{E}$. We also prove that the group of exact auto-equivalences of $\mathrm{D}_{\text {coh }}^{\mathrm{b}}(\boldsymbol{E})$ acts transitively on the set of spherical objects.

In $\S 5$ we study $t$-structures on $\mathrm{D}_{\text {coh }}^{\mathrm{b}}(\boldsymbol{E})$ and stability conditions in the sense of [Bri02]. We completely classify all $t$-structures on this category (Theorem 5.6 ). This allows us to deduce a description of the group of exact auto-equivalences of $\mathrm{D}_{\text {coh }}^{\mathrm{b}}(\boldsymbol{E})$ (Corollary 5.8). As a second application, we calculate Bridgeland's moduli space of stability conditions on $\boldsymbol{E}$ (Proposition 5.12).

The hearts $\mathrm{D}(\theta, \theta+1)$ of the $t$-structures constructed in $\S 5$ are finite-dimensional non-Noetherian Abelian categories of infinite global dimension. In the case of a smooth elliptic curve, this category is equivalent to the category of holomorphic vector bundles on a non-commutative torus in the sense of Polishchuk and Schwarz [PS03, Proposition 3.9]. It is an interesting problem to find such a differential-geometric interpretation of these Abelian categories in the case of singular Weierstraß curves.

Using the technique of Harder-Narasimhan filtrations, we gain new insight into the classification of indecomposable complexes, which was obtained in [BD04b]. It seems plausible that similar methods can be applied to study the derived category of representations of certain derived tame associative algebras, such as gentle algebras, skew-gentle algebras or degenerated tubular algebras; see for example [BD04a]. The study of Harder-Narasimhan filtrations in conjunction with the action of the group of exact auto-equivalences of the derived category should provide new insight into the combinatorics of indecomposable objects in these derived categories.

Notation. We fix an algebraically closed field $\boldsymbol{k}$ of characteristic zero. By $\boldsymbol{E}$ we always denote a Weierstraß curve. This is a reduced irreducible curve of arithmetic genus one, isomorphic to a cubic curve in the projective plane. If not smooth, it has precisely one singular point $s \in \boldsymbol{E}$, which can be a node or a cusp. If $x \in \boldsymbol{E}$ is arbitrary, we denote by $\boldsymbol{k}(x)$ the residue field of $x$ and consider it 


\section{DERIVED CATEGORIES OF IRREDUCIBLE PROJECTIVE CURVES}

as a sky-scraper sheaf supported at $x$. By $\mathrm{D}_{\text {coh }}^{\mathrm{b}}(\boldsymbol{E})$ we denote the derived category of complexes of $\mathcal{O}_{\boldsymbol{E}}$-modules whose cohomology sheaves are coherent and which are non-zero only in finitely many degrees.

\section{Background: the smooth case}

The purpose of this section is to recall well known results about the structure of the bounded derived category of coherent sheaves over a smooth elliptic curve. Proofs of most of these results can be found in [Ati57, Oda71, LM93, Tu93]. The focus of our presentation is on the features and techniques which are essential in the singular case as well. At the end of this section we highlight the main differences between the smooth case and the singular case. It becomes clear that the failure of Serre duality is the main reason why the proofs and even the formulation of some of the main results do not carry over to the singular case. The aim of the subsequent sections will then be to overcome these difficulties, to find correct formulations which generalise to the singular case and to highlight the common features of the bounded derived category in the smooth and singular cases.

With the exception of $\S 2.7$, throughout this section $\boldsymbol{E}$ denotes a smooth elliptic curve over $\boldsymbol{k}$.

\subsection{Homological dimension}

For any two coherent sheaves $\mathcal{F}, \mathcal{G}$ on $\boldsymbol{E}$, Serre duality provides an isomorphism

$$
\operatorname{Ext}^{\nu}(\mathcal{F}, \mathcal{G}) \cong \operatorname{Ext}^{1-\nu}(\mathcal{G}, \mathcal{F})^{*}
$$

This follows from the usual formulation of Serre duality and the fact that any coherent sheaf has a finite locally free resolution. As a consequence, $\operatorname{Ext}^{\nu}(\mathcal{F}, \mathcal{G})=0$ for any $\nu \geqslant 2$, which means that $\operatorname{Coh}_{\boldsymbol{E}}$ has homological dimension one. This implies that any object $X \in \mathrm{D}_{\text {coh }}^{\mathrm{b}}(\boldsymbol{E})$ splits into the direct sum of appropriate shifts of its cohomology sheaves. To see this, start with a complex $X=\left(\mathcal{F}^{-1} \stackrel{f}{\longrightarrow} \mathcal{F}^{0}\right)$ and consider the distinguished triangle in $\mathrm{D}_{\text {coh }}^{\mathrm{b}}(\boldsymbol{E})$

$$
\operatorname{ker}(f)[1] \longrightarrow X \longrightarrow \operatorname{coker}(f) \stackrel{\xi}{\longrightarrow} \operatorname{ker}(f)[2] .
$$

Because $\xi \in \operatorname{Hom}(\operatorname{coker}(f), \operatorname{ker}(f)[2])=\operatorname{Ext}^{2}(\operatorname{coker}(f), \operatorname{ker}(f))=0$, we obtain $X \cong \operatorname{ker}(f)[1] \oplus$ coker $(f)$. Using the same idea we can proceed by induction to get the claim.

\subsection{Indecomposable sheaves are semi-stable}

It is well known that any coherent sheaf $\mathcal{F} \in \mathrm{Coh}_{\boldsymbol{E}}$ has a Harder-Narasimhan filtration

$$
0 \subset \mathcal{F}_{n} \subset \cdots \subset \mathcal{F}_{1} \subset \mathcal{F}_{0}=\mathcal{F}
$$

whose factors $\mathcal{A}_{\nu}:=\mathcal{F}_{\nu} / \mathcal{F}_{\nu+1}$ are semi-stable with decreasing slopes $\mu\left(\mathcal{A}_{n}\right)>\mu\left(\mathcal{A}_{n-1}\right)>\cdots>$ $\mu\left(\mathcal{A}_{0}\right)$. Using the definition of semi-stability, this implies $\operatorname{Hom}\left(\mathcal{A}_{\nu+i}, \mathcal{A}_{\nu}\right)=0$ for all $\nu \geqslant 0$ and $i>0$. Therefore, $\operatorname{Ext}^{1}\left(\mathcal{A}_{0}, \mathcal{F}_{1}\right) \cong \operatorname{Hom}\left(\mathcal{F}_{1}, \mathcal{A}_{0}\right)^{*}=0$, and the exact sequence $0 \rightarrow \mathcal{F}_{1} \rightarrow \mathcal{F} \rightarrow \mathcal{A}_{0} \rightarrow 0$ must split. In particular, if $\mathcal{F}$ is indecomposable, we have $\mathcal{F}_{1}=0$ and $\mathcal{F} \cong \mathcal{A}_{0}$ and $\mathcal{F}$ is semi-stable.

\subsection{Jordan-Hölder factors}

The full sub-category of $\mathrm{Coh}_{\boldsymbol{E}}$ whose objects are the semi-stable sheaves of a fixed slope is an Abelian category in which any object has a Jordan-Hölder filtration with stable factors. If $\mathcal{F}$ and $\mathcal{G}$ are non-isomorphic stable sheaves which have the same slope, we have $\operatorname{Hom}(\mathcal{F}, \mathcal{G})=0$. Based on this fact, in the same way as before, we can deduce that an indecomposable semi-stable sheaf has all its Jordan-Hölder factors isomorphic to each other. 


\section{Burban And B. Kreußler}

\subsection{Simple is stable}

It is well known that any stable sheaf $\mathcal{F}$ is $\operatorname{simple}$, i.e. $\operatorname{Hom}(\mathcal{F}, \mathcal{F}) \cong \boldsymbol{k}$. On a smooth elliptic curve, the converse is true as well, which equips us with a useful homological characterisation of stability.

To see that simple implies stable, we suppose for a contradiction that $\mathcal{F}$ is simple but not stable. This implies the existence of an epimorphism $\mathcal{F} \rightarrow \mathcal{G}$ with $\mathcal{G}$ stable and $\mu(\mathcal{F}) \geqslant \mu(\mathcal{G})$. Serre duality implies $\operatorname{dim} \operatorname{Ext}^{1}(\mathcal{G}, \mathcal{F})=\operatorname{dim} \operatorname{Hom}(\mathcal{F}, \mathcal{G})>0$, hence $\chi(\mathcal{G}, \mathcal{F}):=\operatorname{dim} \operatorname{Hom}(\mathcal{G}, \mathcal{F})-$ $\operatorname{dim} \operatorname{Ext}^{1}(\mathcal{G}, \mathcal{F})<\operatorname{dim} \operatorname{Hom}(\mathcal{G}, \mathcal{F})$. Riemann-Roch gives $\chi(\mathcal{G}, \mathcal{F})=(\mu(\mathcal{F})-\mu(\mathcal{G})) / \operatorname{rk}(\mathcal{F}) \operatorname{rk}(\mathcal{G})>0$, hence $\operatorname{Hom}(\mathcal{G}, \mathcal{F}) \neq 0$. But this produces a non-zero composition $\mathcal{F} \rightarrow \mathcal{G} \rightarrow \mathcal{F}$ which is not an isomorphism, in contradiction to the assumption that $\mathcal{F}$ was simple.

\subsection{Classification}

Atiyah [Ati57] gave a description of all stable sheaves with a fixed slope in the form $\mathcal{E}(r, d) \otimes \mathcal{L}$, where $\mathcal{L}$ is a line bundle of degree zero and $\mathcal{E}(r, d)$ is a particular stable bundle of the fixed slope. The bundle $\mathcal{E}(r, d)$ depends on the choice of a base point $p_{0} \in \boldsymbol{E}$ and its construction reflects the Euclidean algorithm on the pair (rk, deg). We look at this description from a slightly different perspective. We use the twist functors $T_{\mathcal{O}}$ and $T_{\boldsymbol{k}\left(p_{0}\right)}$, which were constructed by Seidel and Thomas [ST01] (see also [Mel97]). They act as equivalences on $\mathrm{D}_{\text {coh }}^{\mathrm{b}}(\boldsymbol{E})$ and, hence, preserve stability.

A stable sheaf of rank $r$ and degree $d$ is sent by $T_{\mathcal{O}}$ to one with $(\mathrm{rk}, \operatorname{deg})$ equal to $(r-d, d)$. If $r<d$ this is a shift of a stable sheaf. The functor $T_{\boldsymbol{k}\left(p_{0}\right)}$ sends the pair $(r, d)$ to $(r, r+d)$ and its inverse sends it to $(r, d-r)$. Therefore, if we follow the Euclidean algorithm, we find a composition of such functors which provides an equivalence between the category of stable sheaves with slope $d / r$ and the category of simple torsion sheaves. Such sheaves are precisely the structure sheaves of closed points $\boldsymbol{k}(x), x \in \boldsymbol{E}$. They are considered to be stable with slope $\infty$.

More generally, this procedure provides an equivalence between the category of semi-stable sheaves of rank $r$ and degree $d$ with the category of torsion sheaves of length equal to $\operatorname{gcd}(r, d)$. This shows, in particular, that the Abelian category of semi-stable sheaves with fixed slope is equivalent to the category of coherent torsion sheaves.

\subsection{Auto-equivalences}

By $\operatorname{Aut}\left(\mathrm{D}_{\text {coh }}^{\mathrm{b}}(\boldsymbol{E})\right)$ we denote the group of all exact auto-equivalences of the triangulated category $\mathrm{D}_{\text {coh }}^{\mathrm{b}}(\boldsymbol{E})$. This group acts on the Grothendieck group $\mathrm{K}(\boldsymbol{E}) \cong \mathrm{K}\left(\mathrm{D}_{\text {coh }}^{\mathrm{b}}(\boldsymbol{E})\right)$. As the kernel of the Chern character is the radical of the Euler form $\langle X, Y\rangle=\operatorname{dim}(\operatorname{Hom}(X, Y))-\operatorname{dim}(\operatorname{Hom}(X, Y[1]))$ which is invariant under this action, it induces an action on the even cohomology $H^{2 *}(\boldsymbol{E}, \mathbb{Z}) \cong \mathbb{Z}^{2}$. Because $\operatorname{dim}(\operatorname{Hom}(\mathcal{F}, \mathcal{G}))>0$ if and only if $\langle\mathcal{F}, \mathcal{G}\rangle>0$, provided $\mathcal{F} \not \mathcal{G}$ are stable sheaves, the induced action on $\mathbb{Z}^{2}$ is orientation preserving. So, we obtain a homomorphism of groups $\varphi: \operatorname{Aut}\left(\mathrm{D}_{\text {coh }}^{\mathrm{b}}(\boldsymbol{E})\right) \rightarrow$ $\mathrm{SL}(2, \mathbb{Z})$, which is surjective because $T_{\mathcal{O}}$ and $T_{\boldsymbol{k}\left(p_{0}\right)}$ are mapped to a pair of generators of $\operatorname{SL}(2, \mathbb{Z})$. Explicitly, if $\mathbb{G}$ is an auto-equivalence, $\varphi(\mathbb{G})$ describes its action on the pair (rk, deg). To understand $\operatorname{ker}(\varphi)$, we observe that $\varphi(\mathbb{G})=\mathbf{1}$ implies that $\mathbb{G}$ sends a simple torsion sheaf $\boldsymbol{k}(x)$ to some $\boldsymbol{k}(y)[2 k]$, because indecomposability is retained. By the same reason, $\mathbb{G}(\mathcal{O})$ is a shifted line bundle of degree zero. However, $\operatorname{Hom}(\mathcal{L}, \boldsymbol{k}(y)[l])=0$, if $\mathcal{L}$ is a line bundle and $l \neq 0$. Hence, after composing $\mathbb{G}$ with a shift, it sends all simple torsion sheaves to simple torsion sheaves, without a shift. Because $\boldsymbol{E}$ is smooth, we can apply a result of Orlov [Orl97] which says that any auto-equivalence $\mathbb{G}$ is a FourierMukai transform [Muk81]. However, any such functor, which sends the sheaves $\boldsymbol{k}(x)$ to torsion sheaves of length one, is of the form $\mathbb{G}(X)=f^{*}(\mathcal{L} \otimes X)$, where $f: \boldsymbol{E} \rightarrow \boldsymbol{E}$ is an automorphism and $\mathcal{L} \in \operatorname{Pic}(\boldsymbol{E})$ a line bundle. Hence, $\operatorname{ker}(\varphi)$ is generated by $\operatorname{Aut}(\boldsymbol{E}), \operatorname{Pic}^{0}(\boldsymbol{E})$ and even shifts. This gives a complete description of the group $\operatorname{Aut}\left(\mathrm{D}_{\mathrm{coh}}^{\mathrm{b}}(\boldsymbol{E})\right)$. A similar approach was used by Lenzing and Meltzer to describe the group of exact auto-equivalences of tubular weighted projective lines [LM00]. 


\section{DERIVED CATEGORIES OF IRREDUCIBLE PROJECTIVE CURVES}

\subsection{Difficulties in the singular case}

Let now $\boldsymbol{E}$ be an irreducible but singular curve of arithmetic genus one. The technical cornerstones of the theory as described in this section fail to be true in this case. More precisely:

(i) the category of coherent sheaves $\mathrm{Coh}_{\boldsymbol{E}}$ has infinite homological dimension;

(ii) there exist indecomposable complexes in $D_{\text {coh }}^{\mathrm{b}}(\boldsymbol{E})$ which are not just shifted sheaves (see $[\mathrm{BD} 04 \mathrm{~b}, \S 3])$

(iii) Serre duality fails to be true in general;

(iv) not all indecomposable vector bundles are semi-stable;

(v) there exist indecomposable coherent sheaves which are neither torsion sheaves nor torsion free sheaves (see [BD04b]).

Most of the trouble is caused by the failure of Serre duality. The basic example is the following. Suppose $s \in \boldsymbol{E}$ is a node, then

$$
\operatorname{Hom}(\boldsymbol{k}(s), \boldsymbol{k}(s)) \cong \boldsymbol{k} \text { and } \operatorname{Ext}^{1}(\boldsymbol{k}(s), \boldsymbol{k}(s)) \cong \boldsymbol{k}^{2} .
$$

Serre duality is available only if at least one of the two sheaves involved has finite homological dimension. This might suggest that replacing $\mathrm{D}_{\text {coh }}^{\mathrm{b}}(\boldsymbol{E})$ by the sub-category of perfect complexes would solve most of the problems (but see Remark 4.10).

In the subsequent sections we overcome these difficulties and point out the similarities between the smooth and the singular cases.

\section{Harder-Narasimhan filtrations}

Throughout this section, $\boldsymbol{E}$ denotes an irreducible reduced projective curve over $\boldsymbol{k}$ of arithmetic genus one. The notion of stability of coherent torsion free sheaves on an irreducible curve is usually defined with the aid of the slope function $\mu(\cdot)=\operatorname{deg}(\cdot) / \operatorname{rk}(\cdot)$. To use the phase function instead is equivalent, but better adapted for the generalisation to derived categories described below. By definition, the phase $\varphi(\mathcal{F})$ of a non-zero coherent sheaf $\mathcal{F}$ is the unique number which satisfies $0<\varphi(\mathcal{F}) \leqslant 1$ and $m(\mathcal{F}) \exp (\pi i \varphi(\mathcal{F}))=-\operatorname{deg}(\mathcal{F})+i \operatorname{rk}(\mathcal{F})$, where $m(\mathcal{F})$ is a positive real number, called the mass of the sheaf $\mathcal{F}$. In particular, $\varphi(\mathcal{O})=1 / 2$ and all non-zero torsion sheaves have phase one. A torsion free coherent sheaf $\mathcal{F}$ is called semi-stable if, for any exact sequence of torsion free coherent sheaves

$$
0 \longrightarrow \mathcal{E} \longrightarrow \mathcal{F} \longrightarrow \mathcal{G} \longrightarrow 0
$$

the inequality $\varphi(\mathcal{E}) \leqslant \varphi(\mathcal{F})$, or equivalently $\varphi(\mathcal{F}) \leqslant \varphi(\mathcal{G})$, holds. It is well known [Rud97] that any torsion free coherent sheaf $\mathcal{F}$ on a projective variety has a Harder-Narasimhan filtration

$$
0 \subset \mathcal{F}_{n} \subset \mathcal{F}_{n-1} \subset \cdots \subset \mathcal{F}_{1} \subset \mathcal{F}_{0}=\mathcal{F}
$$

which is uniquely characterised by the property that all factors $\mathcal{A}_{i}=\mathcal{F}_{i} / \mathcal{F}_{i+1}$ are semi-stable and satisfy

$$
\varphi\left(\mathcal{A}_{n}\right)>\varphi\left(\mathcal{A}_{n-1}\right)>\cdots>\varphi\left(\mathcal{A}_{0}\right) .
$$

Originally, this concept of stability was introduced in the 1960s in order to construct moduli spaces using geometric invariant theory. It could also be seen as a method to understand the structure of the category of coherent sheaves on a projective variety. By Simpson, the notion of stability was extended to coherent sheaves of pure dimension. A very general approach was taken by Rudakov [Rud97], who introduced the notion of stability on Abelian categories. Under some finiteness assumptions on the category, he shows the existence and uniqueness of a Harder-Narasimhan filtration for any object of the category in question. As an application of his work, the usual slope 


\section{Burban and B. KreußleR}

stability extends to the whole category $\mathrm{Coh}_{\boldsymbol{E}}$ of coherent sheaves on $\boldsymbol{E}$. In particular, any non-zero coherent sheaf has a Harder-Narasimhan filtration and any non-zero coherent torsion sheaf on the curve $\boldsymbol{E}$ is semi-stable.

Inspired by work of Douglas on П-stability for D-branes (see for example [Dou02]), it was shown by Bridgeland [Bri02] how to extend the concept of stability and Harder-Narasimhan filtration to the derived category of coherent sheaves, or more generally to a triangulated category. These new ideas were merged with the ideas from [Rud97] in the paper [GKR04]. We shall follow here the approach of Bridgeland [Bri02]. In $\S 5$ we give a description of Bridgeland's moduli space of stability conditions on the derived category of irreducible singular curves of arithmetic genus one. However, throughout the present chapter we stick to the classical notion of stability on the category of coherent sheaves and the stability structure it induces on the triangulated category.

In order to generalise the concept of a Harder-Narasimhan filtration to the category $\mathrm{D}_{\text {coh }}^{\mathrm{b}}(\boldsymbol{E})$, Bridgeland [Bri02] extends the definition of the phase of a sheaf to shifts of coherent sheaves by

$$
\varphi(\mathcal{F}[n]):=\varphi(\mathcal{F})+n,
$$

where $\mathcal{F} \neq 0$ is a coherent sheaf on $\boldsymbol{E}$ and $n \in \mathbb{Z}$. A complex which is non-zero at position $m$ only has, according to this definition, phase in the interval $(-m,-m+1]$. If $\mathcal{F}$ and $\mathcal{F}^{\prime}$ are non-zero coherent sheaves and $a, b$ are integers, we have the implication

$$
\varphi(\mathcal{F}[-a])>\varphi\left(\mathcal{F}^{\prime}[-b]\right) \Longrightarrow a \leqslant b .
$$

For any $\varphi \in \mathbb{R}$ we denote by $\mathrm{P}(\varphi)$ the Abelian category of shifted semi-stable sheaves with phase $\varphi$. Of course, $0 \in \mathrm{P}(\varphi)$ for all $\varphi$. If $\varphi \in(0,1]$, this is a full Abelian subcategory of $\mathrm{Coh}_{\boldsymbol{E}}$. For any $\varphi \in \mathbb{R}$ we have $\mathrm{P}(\varphi+n)=\mathrm{P}(\varphi)[n]$. A non-zero object of $\mathrm{D}_{\text {coh }}^{\mathrm{b}}(\boldsymbol{E})$ will be called semi-stable if it is an element of one of the categories $\mathrm{P}(\varphi), \varphi \in \mathbb{R}$.

Bridgeland's stability conditions [Bri02] involve so-called central charges. In order to define the central charge of the standard stability condition, we need a definition of degree and rank for arbitrary objects in $\mathrm{D}_{\text {coh }}^{\mathrm{b}}(\boldsymbol{E})$.

Let $K=\mathcal{O}_{\boldsymbol{E}, \eta}$ be the field of rational functions on the irreducible curve $\boldsymbol{E}$ with generic point $\eta \in \boldsymbol{E}$. The base change $\eta: \operatorname{Spec}(K) \rightarrow \boldsymbol{E}$ is flat, so that $\eta^{*}(F)$, taken in the non-derived sense, is correctly defined for any $F \in \mathrm{D}_{\text {coh }}^{\mathrm{b}}(\boldsymbol{E})$. We define $\operatorname{rk}(F):=\chi\left(\eta^{*}(F)\right)$, which is the alternating sum of the dimensions of the cohomology spaces of the complex $\eta^{*}(F)$ which are vector spaces over $K$.

In order to define the degree, we use the functor

$$
\boldsymbol{R} \operatorname{Hom}\left(\mathcal{O}_{\boldsymbol{E}}, \cdot\right): \mathrm{D}_{\mathrm{coh}}^{\mathrm{b}}(\boldsymbol{E}) \longrightarrow \mathrm{D}_{\text {coh }}^{\mathrm{b}}(\boldsymbol{k}),
$$

and set $\operatorname{deg}(F):=\chi\left(\boldsymbol{R} \operatorname{Hom}\left(\mathcal{O}_{\boldsymbol{E}}, F\right)\right)$. Here, we denoted by $\mathrm{D}_{\text {coh }}^{\mathrm{b}}(\boldsymbol{k})$ the bounded derived category of finite-dimensional vector spaces over $\boldsymbol{k}$. For coherent sheaves, these definitions coincide with the usual definitions of rank and degree. In particular, a torsion sheaf of length $m$ which is supported at a single point of $\boldsymbol{E}$ has rank 0 and degree $m$.

These definitions imply that rank and degree are additive on distinguished triangles in $D_{\text {coh }}^{\mathrm{b}}(\boldsymbol{E})$. Hence, they induce homomorphisms on the Grothendieck group $\mathrm{K}\left(\mathrm{D}_{\text {coh }}^{\mathrm{b}}(\boldsymbol{E})\right)$ of the triangulated category $\mathrm{D}_{\text {coh }}^{\mathrm{b}}(\boldsymbol{E})$, which is by definition the quotient of the free Abelian group generated by the objects of $\mathrm{D}_{\text {coh }}^{\mathrm{b}}(\boldsymbol{E})$ modulo expressions coming from distinguished triangles. Recall that $\mathrm{K}_{0}(\mathrm{Coh}(\boldsymbol{E})) \cong$ $\mathrm{K}\left(\mathrm{D}_{\text {coh }}^{\mathrm{b}}(\boldsymbol{E})\right)$; see [Gro77]. We denote this group by $\mathrm{K}(\boldsymbol{E})$.

LEMmA 3.1. If $\boldsymbol{E}$ is an irreducible singular curve of arithmetic genus one, we have $\mathrm{K}(\boldsymbol{E}) \cong \mathbb{Z}^{2}$ with generators $[\boldsymbol{k}(x)]$ and $\left[\mathcal{O}_{\boldsymbol{E}}\right]$.

Proof. Recall that the Grothendieck-Riemann-Roch theorem (see [BFM75] or [Ful89]) provides a homomorphism

$$
\tau_{\boldsymbol{E}}: \mathrm{K}(\boldsymbol{E}) \longrightarrow A_{*}(\boldsymbol{E}) \otimes \mathbb{Q}
$$




\section{DERIVED CATEGORIES OF IRREDUCIBLE PROJECTIVE CURVES}

which depends functorially on $\boldsymbol{E}$ with respect to proper direct images. Moreover, $\left(\tau_{\boldsymbol{E}}\right)_{\mathbb{Q}}: \mathrm{K}(\boldsymbol{E}) \otimes \mathbb{Q} \rightarrow$ $A_{*}(\boldsymbol{E}) \otimes \mathbb{Q}$ is an isomorphism (see [Ful89, Corollary 18.3.2]).

If $\boldsymbol{E}$ is an irreducible singular projective curve of arithmetic genus one, we easily see that the Chow group $A_{*}(\boldsymbol{E})$ is isomorphic to $\mathbb{Z}^{2}$. The two generators are $[x] \in A_{0}(\boldsymbol{E})$ with $x \in \boldsymbol{E}$ and $[\boldsymbol{E}] \in A_{1}(\boldsymbol{E})$. Note that $[x]=[y] \in A_{0}(\boldsymbol{E})$ for any two closed points $x, y \in \boldsymbol{E}$, because the normalisation of $\boldsymbol{E}$ is $\mathbb{P}^{1}$. Using [Ful89, Theorem $18.3(5)$ ], we obtain $\tau_{\boldsymbol{E}}(\boldsymbol{k}(x))=[x] \in A_{0}(\boldsymbol{E})$ for any $x \in \boldsymbol{E}$. On the other hand, from [Ful89, Example 18.3.4 (a)], we obtain $\tau_{\boldsymbol{E}}\left(\mathcal{O}_{\boldsymbol{E}}\right)=[\boldsymbol{E}] \in A_{1}(\boldsymbol{E})$. Therefore, the classes of $\boldsymbol{k}(x)$ and $\mathcal{O}_{\boldsymbol{E}}$ define a basis of $\mathrm{K}(\boldsymbol{E}) \otimes \mathbb{Q}$. However, these two classes generate the group $\mathrm{K}(\boldsymbol{E})$, so that it must be a free Abelian group.

The central charge of the standard stability structure on $\mathrm{D}_{\text {coh }}^{\mathrm{b}}(\boldsymbol{E})$ is the homomorphism of Abelian groups

which is given by

$$
Z: \mathrm{K}(\boldsymbol{E}) \longrightarrow \mathbb{Z} \oplus i \mathbb{Z} \subset \mathbb{C}
$$

$$
Z(F):=-\operatorname{deg}(F)+i \operatorname{rk}(F) .
$$

If $F$ is a non-zero coherent sheaf, $Z(F)$ is a point on the ray from the origin through $\exp (\pi i \varphi(F))$ in $\mathbb{C}$. Its distance from the origin was called the mass of $F$.

Although the phase $\varphi(F)$ is defined for sheaves and their shifts only, we are able to define the slope $\mu(F)$ for any object in $\mathrm{D}_{\text {coh }}^{\mathrm{b}}(\boldsymbol{E})$ which is not equal to zero in the Grothendieck group. Namely, the usual definition $\mu(F):=\operatorname{deg}(F) / \operatorname{rk}(F)$ gives us now a mapping

$$
\mu: \mathrm{K}(\boldsymbol{E}) \backslash\{0\} \longrightarrow \mathbb{Q} \cup\{\infty\},
$$

which extends the usual definition of the slope of a sheaf. Because $Z\left(\mathcal{O}_{\boldsymbol{E}}\right)=i$ and $Z(\boldsymbol{k}(x))=-1$, Lemma 3.1 implies that $Z$ is injective. Therefore, $\mu$ is defined for any non-zero element of the Grothendieck group.

For arbitrary objects $X \in \mathrm{D}_{\text {coh }}^{\mathrm{b}}(\boldsymbol{E})$ we have $Z(X[1])=-Z(X)$, hence $\mu(X[1])=\mu(X)$ when defined. In the case of shifted sheaves, in contrast to the slope $\mu$, the phase $\varphi$ keeps track of the position of this sheaf in the complex. As an illustration, we include an example of an indecomposable object in $\mathrm{D}_{\text {coh }}^{\mathrm{b}}(\boldsymbol{E})$ which has a zero image in the Grothendieck group.

Example 3.2. Let $s \in \boldsymbol{E}$ be the singular point and denote, as usual, by $\boldsymbol{k}(s)$ the torsion sheaf of length one which is supported at $s$. This sheaf does not have finite homological dimension. To see this, we observe first that $\operatorname{Ext}^{k}(\boldsymbol{k}(s), \boldsymbol{k}(s)) \cong H^{0}\left(\mathcal{E} x t^{k}(\boldsymbol{k}(s), \boldsymbol{k}(s))\right)$. Moreover, as an $\mathcal{O}_{\boldsymbol{E}, s^{-}}$module, $\boldsymbol{k}(s)$ has an infinite periodic locally free resolution of the form

$$
\cdots \stackrel{A}{\longrightarrow} \mathcal{O}_{\boldsymbol{E}, s}^{2} \stackrel{B}{\longrightarrow} \mathcal{O}_{\boldsymbol{E}, s}^{2} \stackrel{A}{\longrightarrow} \mathcal{O}_{\boldsymbol{E}, s}^{2} \longrightarrow \mathcal{O}_{\boldsymbol{E}, s} \longrightarrow \boldsymbol{k}(s) \longrightarrow 0,
$$

where $A B=B A=f \cdot I_{2}$ is a reduced matrix factorisation of an equation $f$ of $\boldsymbol{E} \subset \mathbb{P}^{2}$. For example, if $s$ is a node, so that $\boldsymbol{E}$ is locally given by the polynomial $f=y^{2}-x^{3}-x^{2} \in \boldsymbol{k}[x, y]$, we can choose

$$
A=\left(\begin{array}{cc}
y & x^{2}+x \\
x & y
\end{array}\right) \text { and } B=\left(\begin{array}{cc}
y & -x^{2}-x \\
-x & y
\end{array}\right)
$$

considered modulo $f$. More generally, any singular Weierstraß cubic $f$ can be written as $y \cdot y-R \cdot S$ with $y, R, S$ all vanishing at the singular point. The off-diagonal elements of $A$ and $B$ are then formed by $\pm R, \pm S$. Therefore, all entries of the matrices $A$ and $B$ are elements of the maximal ideal of the local ring $\mathcal{O}_{\boldsymbol{E}, s}$. Hence, the application of $\operatorname{Hom}(\cdot, \boldsymbol{k}(s))$ produces a complex with zero differential, which implies that $\operatorname{Ext}^{k}(\boldsymbol{k}(s), \boldsymbol{k}(s))$ is two-dimensional for all $k \geqslant 1$. In particular, $\operatorname{Ext}^{2}(\boldsymbol{k}(s), \boldsymbol{k}(s)) \cong \boldsymbol{k}^{2}$, and we can pick a non-zero element $w \in \operatorname{Hom}(\boldsymbol{k}(s), \boldsymbol{k}(s)[2])$. There exists a 


\section{Burban AND B. Kreußler}

complex $X \in \mathrm{D}_{\text {coh }}^{\mathrm{b}}(\boldsymbol{E})$ which sits in a distinguished triangle

$$
X \longrightarrow \boldsymbol{k}(s) \stackrel{w}{\longrightarrow} \boldsymbol{k}(s)[2] \stackrel{+}{\longrightarrow} .
$$

Because the shift by one corresponds to multiplication by -1 in the Grothendieck group, this object $X$ is equal to zero in $\mathrm{K}(\boldsymbol{E})$. On the other hand, $X$ is indecomposable. Indeed, if $X$ would split, it must be $X \cong \boldsymbol{k}(s) \oplus \boldsymbol{k}(s)[1]$, because the only non-zero cohomology of $X$ is $H^{-1}(X) \cong \boldsymbol{k}(s)$ and $H^{0}(X) \cong \boldsymbol{k}(s)$. However, because $\operatorname{Hom}(\boldsymbol{k}(s)[1], \boldsymbol{k}(s))=0$, Lemma 3.11, applied to the distinguished triangle

$$
\boldsymbol{k}(s)[1] \longrightarrow X \longrightarrow \boldsymbol{k}(s) \stackrel{+}{w}
$$

with $X \cong \boldsymbol{k}(s) \oplus \boldsymbol{k}(s)[1]$, implies $w=0$.

Definition 3.3 [Bri02]. A Harder-Narasimhan filtration (HNF) of an object $X \in \mathrm{D}_{\text {coh }}^{\mathrm{b}}(\boldsymbol{E})$ is a finite collection of distinguished triangles

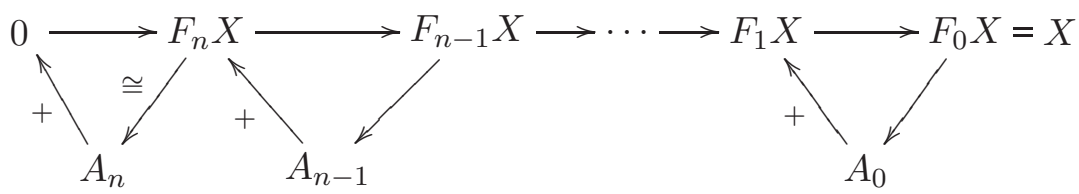

with $A_{j} \in \mathrm{P}\left(\varphi_{j}\right)$ and $A_{j} \neq 0$ for all $j$, such that $\varphi_{n}>\varphi_{n-1}>\cdots>\varphi_{0}$.

If all ingredients of an HNF are shifted by one, we obtain an HNF of $X$ [1]. The shifted sheaves $A_{j}$ are called the semi-stable HN-factors of $X$ and we define $\varphi_{+}(X):=\varphi_{n}$ and $\varphi_{-}(X):=\varphi_{0}$. Later, Theorem 3.10, we show that the HNF of an object $X$ is unique up to isomorphism. This justifies this notation. For the moment, we keep in mind that $\varphi_{+}(X)$ and $\varphi_{-}(X)$ might depend on the HNF and not only on the object $X$.

Before we proceed, we include a few remarks about the notation we use. Distinguished triangles in a triangulated category are displayed as either

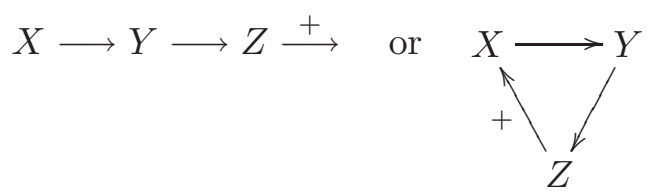

where the arrow which is marked with + is in fact a morphism $Z \rightarrow X[1]$.

We shall use the octahedron axiom, the axiom (TR4) in Verdier's list, in the following convenient form: If two morphisms $X \stackrel{f}{\longrightarrow} Y \stackrel{g}{\longrightarrow} Z$ are given, for any three distinguished triangles with bases $f, g$ and $g \circ f$ there exists a fourth distinguished triangle which is indicated below by dashed arrows, such that we obtain the following commutative diagram.

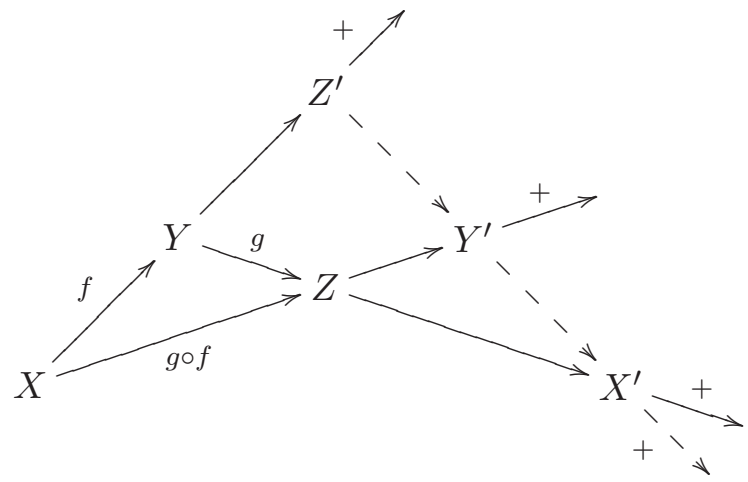

1238 


\section{DERIVED CATEGORIES OF IRREDUCIBLE PROJECTIVE CURVES}

The remainder of this section is devoted to the proofs of the crucial properties of HarderNarasimhan filtrations in triangulated categories. These properties can be found in [Bri02, GKR04], where most of them appear to be either implicit or without a detailed proof.

LEMMA 3.4. Let

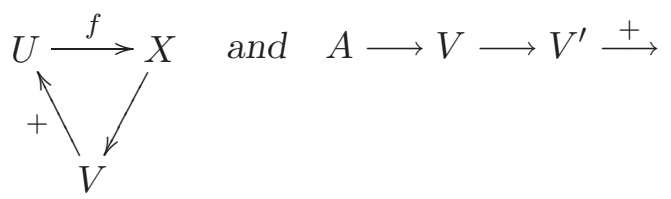

be distinguished triangles. Then there exist a factorisation $U \longrightarrow W \stackrel{f^{\prime}}{\longrightarrow} X$ of $f$ and the following two distinguished triangles.

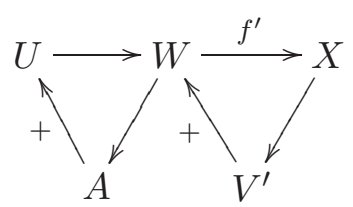

Proof. If we apply the octahedron axiom to the composition $A \rightarrow V \rightarrow U[1]$ we obtain the commutative diagram

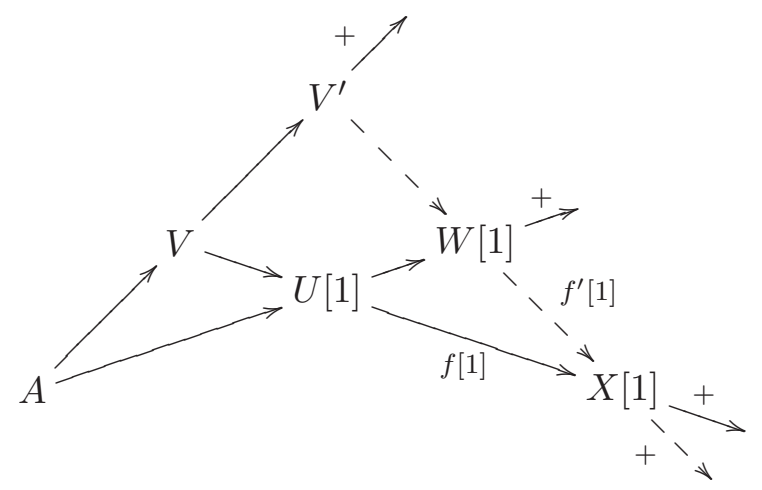

which gives the claim.

LEMMA 3.5. Let

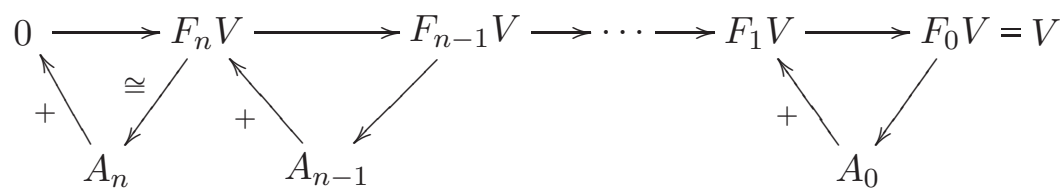

be an HNF of $V \in \mathrm{D}_{\text {coh }}^{\mathrm{b}}(\boldsymbol{E})$ and $F_{k} V \longrightarrow V \longrightarrow V^{\prime} \stackrel{+}{\longrightarrow}$ a distinguished triangle with $1 \leqslant$ $k \leqslant n$. Then, $F_{k} V$ has an HNF with $H N$-factors $A_{n}, A_{n-1}, \ldots, A_{k}$ and $V^{\prime}$ one with $H N$-factors $A_{k-1}, A_{k-2}, \ldots, A_{0}$.

Proof. The first statement is clear, because we can cut off the HNF of $V$ at $F_{k} V$ to obtain an HNF of $F_{k} V$. Let us define objects $F_{i} V^{\prime}$ by exact triangles $F_{k} V \longrightarrow F_{i} V \longrightarrow F_{i} V^{\prime} \stackrel{+}{\longrightarrow}$, where the first arrow is the composition of the morphisms in the HNF of $V$. Using the octahedron axiom, we obtain 


\section{Burban and B. Kreußler}

for any $i \leqslant k$ the commutative diagram

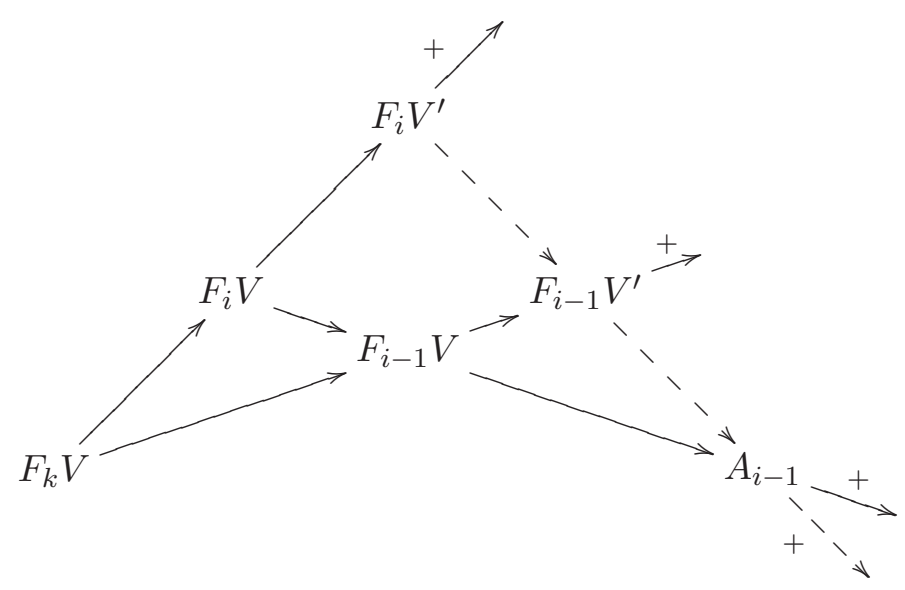

which implies the second claim.

Remark 3.6. The statement of Lemma 3.5 is true with identical proof if we relax the assumption of being an HNF by allowing $\varphi\left(A_{k}\right)=\varphi\left(A_{k-1}\right)$ for the chosen value of $k$.

LEMMA 3.7. If

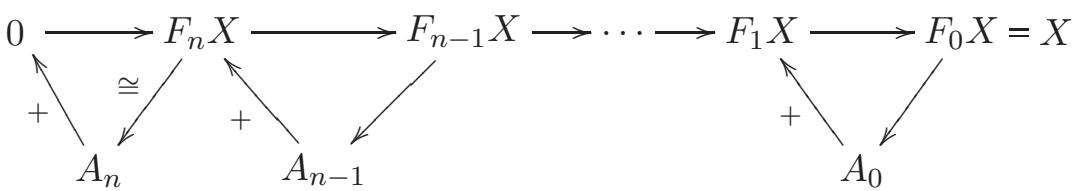

is an HNF of $X \in \mathrm{D}_{\mathrm{coh}}^{\mathrm{b}}(\boldsymbol{E})$ such that $A_{0}[k]$ is a sheaf, then $H^{k}(X) \neq 0$. In particular, the following implication is true:

$$
X \in \mathrm{D}^{\leqslant m} \Longrightarrow \forall i \geqslant 0: A_{i} \in \mathrm{D}^{\leqslant m} .
$$

Proof. The assumption $A_{0}[k] \in \operatorname{Coh}_{\boldsymbol{E}}$ means $H^{k}\left(A_{0}\right)=A_{0}[k] \neq 0$ and $\varphi\left(A_{0}\right) \in(-k,-k+1]$. Because for all $i>0$ we have $\varphi\left(A_{i}\right)>\varphi\left(A_{0}\right)$, we obtain $\varphi\left(A_{i}\right)>-k$. This implies $H^{k+1}\left(A_{i}\right)=0$ for all $i \geqslant 0$. The cohomology sequences of the distinguished triangles $F_{i+1} \longrightarrow F_{i} \longrightarrow A_{i} \stackrel{+}{\longrightarrow}$ imply $H^{k+1}\left(F_{i} X\right)=0$ for all $i>0$ and an exact sequence $H^{k}(X) \rightarrow H^{k}\left(A_{0}\right) \rightarrow H^{k+1}\left(F_{1} X\right)$, hence $H^{k}(X) \neq 0$. The statement about the other HN-factors $A_{i}$ follows now from $\varphi\left(A_{i}\right) \geqslant \varphi\left(A_{0}\right)$.

Proposition 3.8. Any non-zero object $X \in \mathrm{D}_{\text {coh }}^{\mathrm{b}}(\boldsymbol{E})$ has an HNF.

Proof. The existence of an HNF for objects of $\mathrm{Coh}_{\boldsymbol{E}}$ is classically known; see [HN75, Rud97]. Therefore, we can proceed by induction on the number of non-zero cohomology sheaves of $X \in \mathrm{D}_{\text {coh }}^{\mathrm{b}}(\boldsymbol{E})$. If $n$ is the largest integer with $H^{n}(X) \neq 0$, we have a distinguished triangle

$$
\tau^{\leqslant n-1} X \longrightarrow X \longrightarrow H^{n}(X)[-n] \stackrel{+}{\longrightarrow} .
$$

By inductive hypothesis, there exists an HNF of $\tau^{\leqslant n-1} X$. From Lemma 3.7 we conclude that all HN-factors of $\tau^{\leqslant n-1} X$ are in $\mathrm{D}^{\leqslant n-1}$ and so $\varphi_{-}\left(\tau^{\leqslant n-1} X\right)>-n+1$.

Because $H^{n}(X)$ is a sheaf, we have $\varphi_{+}\left(H^{n}(X)[-n]\right) \in(-n,-n+1]$, hence $\varphi_{-}\left(\tau^{\leqslant n-1} X\right)>$ $\varphi_{+}\left(H^{n}(X)[-n]\right)$.

We prove now for any distinguished triangle

$$
U \longrightarrow X \longrightarrow V \stackrel{+}{\longrightarrow}
$$

in which $V[n]$ is a coherent sheaf that the existence of an HNF for $U$ with $\varphi_{-}(U)>\varphi_{+}(V)$ implies the existence of an HNF of $X$. 


\section{DERIVED CATEGORIES OF IRREDUCIBLE PROJECTIVE CURVES}

Because $V[n]$ is a sheaf, $V$ has an HNF and we proceed by induction on the number of HN-factors of $V$. Let $A$ be the leftmost object in an HNF of $V$, i.e. $A \in \mathrm{P}\left(\varphi_{+}(V)\right)$. By Lemma 3.4 applied to the distinguished triangles $(2)$ and $A \longrightarrow V \longrightarrow V^{\prime} \stackrel{+}{\longrightarrow}$, there exist two distinguished triangles in which $V^{\prime}[n]$ is a coherent sheaf with a smaller number of HN-factors as $V$ :

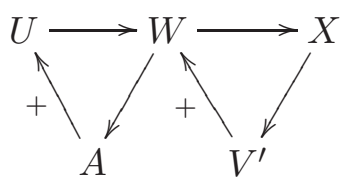

Because $\varphi_{-}(U) \geqslant \varphi(A)=\varphi_{+}(V)$, the left triangle can be concatenated to the given HNF of $U$ in order to provide an HNF for $W$. The start of the induction is covered as well: it is the case $V^{\prime}=0$.

Lemma 3.9. If $X, Y \in \mathrm{D}_{\text {coh }}^{\mathrm{b}}(\boldsymbol{E})$ with $\varphi_{-}(X)>\varphi_{+}(Y)$, then

$$
\operatorname{Hom}(X, Y)=0 \text {. }
$$

Proof. If $X, Y$ are semi-stable sheaves, this is well known and follows easily from the definition of semi-stability. Because $\operatorname{Hom}(X, Y[k])=0$, if $X, Y$ are sheaves and $k<0$, the claim follows if $X \in \mathrm{P}(\varphi)$ and $Y \in \mathrm{P}(\psi)$ with $\varphi>\psi$. Let now $X \in \mathrm{P}(\varphi)$ and $Y \in \mathrm{D}_{\text {coh }}^{\mathrm{b}}(\boldsymbol{E})$ with $\varphi>\varphi_{+}(Y)$. Let

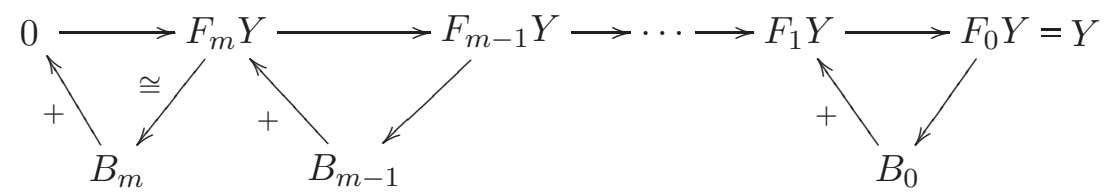

be an HNF of $Y$. We have $\varphi\left(B_{j}\right) \leqslant \varphi\left(B_{m}\right)=\varphi_{+}(Y)$, hence $\varphi(X)>\varphi\left(B_{j}\right)$ and $\operatorname{Hom}\left(X, B_{j}\right)=0$ for all $j$. If we apply the functor $\operatorname{Hom}(X, \cdot)$ to the distinguished triangles $F_{j+1} Y \longrightarrow F_{j} Y \longrightarrow B_{j} \stackrel{+}{\longrightarrow}$, we obtain surjections $\operatorname{Hom}\left(X, F_{j+1} Y\right) \rightarrow \operatorname{Hom}\left(X, F_{j} Y\right)$. From $\operatorname{Hom}\left(X, F_{m} Y\right)=\operatorname{Hom}\left(X, B_{m}\right)=0$, we obtain $\operatorname{Hom}(X, Y)=\operatorname{Hom}\left(X, F_{0} Y\right)=0$.

Let now $X, Y$ be arbitrary non-zero objects of $\mathrm{D}_{\text {coh }}^{\mathrm{b}}(\boldsymbol{E})$ which satisfy $\varphi_{-}(X)>\varphi_{+}(Y)$. If

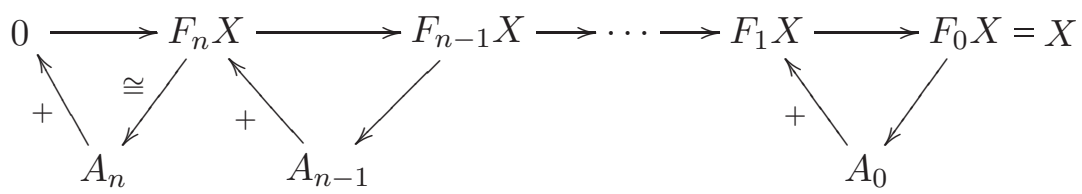

is an HNF of $X$, we have $\varphi\left(A_{i}\right) \geqslant \varphi\left(A_{0}\right)=\varphi_{-}(X)>\varphi_{+}(Y)$. We know already that $\operatorname{Hom}\left(A_{i}, Y\right)=0$ for all $i \geqslant 0$. If we apply the functor $\operatorname{Hom}(\cdot, Y)$ to the distinguished triangles $F_{i+1} X \longrightarrow F_{i} X \longrightarrow$ $A_{i} \stackrel{+}{\longrightarrow}$, we obtain injections $\operatorname{Hom}\left(F_{i} X, Y\right) \hookrightarrow \operatorname{Hom}\left(F_{i+1} X, Y\right)$. Again, this implies $\operatorname{Hom}(X, Y)=$ 0 .

Theorem 3.10 [Bri02, GKR04]. The HNF of any non-zero object $X \in \mathrm{D}_{\mathrm{coh}}^{\mathrm{b}}(\boldsymbol{E})$ is unique up to unique isomorphism.

Proof. If

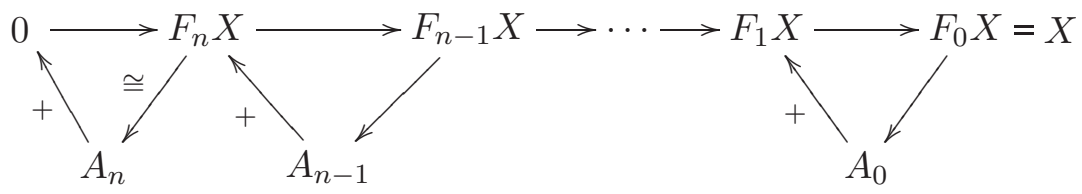




\section{Burban And B. Kreußler}

and

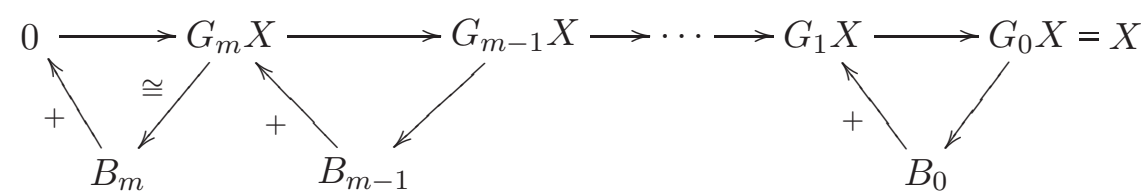

are HNFs of $X$, we have to show that there exist unique isomorphisms of distinguished triangles for any $k \geqslant 0$

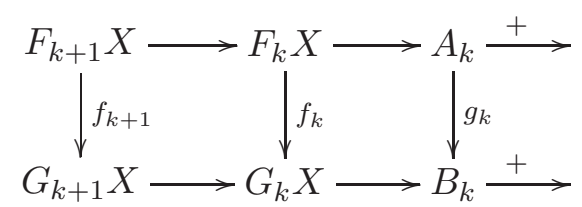

with $f_{0}=\operatorname{ld}_{X}$. This is obtained by induction on $k \geqslant 0$ from the following claim: If an isomorphism $f: F \rightarrow G$ and two distinguished triangles $F^{\prime} \longrightarrow F \longrightarrow A \stackrel{+}{\longrightarrow}$ and $G^{\prime} \longrightarrow G \longrightarrow B \stackrel{+}{\longrightarrow}$ are given such that $A \in \mathrm{P}(\varphi), B \in \mathrm{P}(\psi)$ and $F^{\prime}, G^{\prime}$ have HNFs with $\varphi_{-}\left(F^{\prime}\right)>\varphi$ and $\varphi_{-}\left(G^{\prime}\right)>\psi$, then there exist unique isomorphisms $f^{\prime}: F^{\prime} \rightarrow G^{\prime}$ and $g: A \rightarrow B$ such that $\left(f^{\prime}, f, g\right)$ is a morphism of triangles. In particular, $\varphi=\psi$.

Without loss of generality, we may assume $\varphi \geqslant \psi$. This implies $\varphi_{-}\left(F^{\prime}[1]\right)>\varphi_{-}\left(F^{\prime}\right)>\psi$. Lemma 3.9 implies therefore that $\operatorname{Hom}\left(F^{\prime}, B\right)=\operatorname{Hom}\left(F^{\prime}[1], B\right)=0$. From [BBD82, Proposition 1.1.9], we obtain the existence and uniqueness of the morphisms $f^{\prime}, g$. It remains to show that they are isomorphisms. If $g$ were zero, the second morphism in the triangle $G^{\prime} \longrightarrow G \stackrel{0}{\longrightarrow} B \stackrel{+}{\longrightarrow}$ would be zero. Hence, $B$ would be a direct summand of $G^{\prime}[1]$ which implies $\operatorname{Hom}\left(G^{\prime}[1], B\right) \neq 0$. This contradicts Lemma 3.9, because $\varphi_{-}\left(G^{\prime}[1]\right)>\varphi\left(G^{\prime}\right)>\psi=\varphi(B)$. Hence, $g \neq 0$ and Lemma 3.9 implies $\varphi(A) \leqslant \varphi(B)$, i.e. $\varphi=\psi$. So, the same reasoning as before gives a unique morphism of distinguished triangles in the other direction. The composition of both are the respective identities of $F^{\prime} \longrightarrow F \longrightarrow A \stackrel{+}{\longrightarrow}$ and $G^{\prime} \longrightarrow G \longrightarrow B \stackrel{+}{\longrightarrow}$ respectively, which follows again from the uniqueness part of [BBD82, Proposition 1.1.9]. This proves the claim.

We need the following useful lemma.

Lemma 3.11 [PX97, Lemma 2.5]. Let D be a triangulated category and let

$$
F \longrightarrow G \longrightarrow H_{1} \oplus H_{2} \underset{(0, w)}{\stackrel{+}{\longrightarrow}}
$$

be a distinguished triangle in $\mathrm{D}$. Then $G \cong H_{1} \oplus G^{\prime}$ splits and the given triangle is isomorphic to the following:

$$
F \stackrel{\left(\begin{array}{l}
0 \\
g
\end{array}\right)}{\longrightarrow} H_{1} \oplus G^{\prime} \stackrel{\left(\begin{array}{ll}
1 & 0 \\
0 & f^{\prime}
\end{array}\right)}{\longrightarrow} H_{1} \oplus H_{2} \stackrel{+}{(0, w)}
$$

Dually, if

$$
F \stackrel{\left(\begin{array}{l}
0 \\
g
\end{array}\right)}{\longrightarrow} G_{1} \oplus G_{2} \longrightarrow H \stackrel{+}{\longrightarrow}
$$

is a distinguished triangle then $H \cong G_{1} \oplus H^{\prime}$ and the given triangle is isomorphic to the following:

$$
F \stackrel{\left(\begin{array}{l}
0 \\
g
\end{array}\right)}{\longrightarrow} G_{1} \oplus G_{2} \stackrel{\left(\begin{array}{ll}
1 & 0 \\
0 & f^{\prime}
\end{array}\right)}{\longrightarrow} G_{1} \oplus H^{\prime} \stackrel{+}{(0, w)} .
$$

The results in this section are true for more general triangulated categories than $\mathrm{D}_{\text {coh }}^{\mathrm{b}}(\boldsymbol{E})$. Without changes, the proofs apply if we replace $\mathrm{D}_{\text {coh }}^{\mathrm{b}}(\boldsymbol{E})$ by the bounded derived category of an Abelian 


\section{DERIVED CATEGORIES OF IRREDUCIBLE PROJECTIVE CURVES}

category which is equipped with the notion of stability in the sense of [Rud97]. In particular, these results hold for polynomial stability on the triangulated categories $\mathrm{D}_{\text {coh }}^{\mathrm{b}}(X)$ where $X$ is a projective variety over $\boldsymbol{k}$.

\section{The structure of the bounded derived category of coherent sheaves on a singular Weierstraß curve}

In this section, we prove the main results on which our understanding of $D_{\text {coh }}^{\text {b }}(\boldsymbol{E})$ is based. Again, $\boldsymbol{E}$ denotes a Weierstraß curve. Our main focus is on the singular case, but all the results remain true in the smooth case as well. A speciality of this category is the non-vanishing result, Proposition 4.12. Unlike the smooth case, there exist indecomposable objects in $\mathrm{D}_{\text {coh }}^{\mathrm{b}}(\boldsymbol{E})$, which are not semi-stable. Their Harder-Narasimhan factors are characterised in Proposition 4.6. We propose to visualise indecomposable objects by their 'shadows'. As an application of our results, we give a complete characterisation of all spherical objects in $\mathrm{D}_{\text {coh }}^{\mathrm{b}}(\boldsymbol{E})$. As a consequence, we show that the group of exact auto-equivalences acts transitively on the set of spherical objects. This answers a question which was posed by Polishchuk [Pol02].

Let us set up some notation. For any $\varphi \in(0,1]$ we denote by $\mathrm{P}(\varphi)^{s} \subset \mathrm{P}(\varphi)$ the full sub-category of stable sheaves with phase $\varphi$. We extend this definition to all $\varphi \in \mathbb{R}$ by requiring $\mathrm{P}(\varphi+n)^{s}=\mathrm{P}(\varphi)^{s}[n]$ for all $n \in \mathbb{Z}$ and all $\varphi \in \mathbb{R}$.

We already know the structure of $\mathrm{P}(1)^{s}$. Because $\mathrm{P}(1)$ is the category of coherent torsion sheaves on $\boldsymbol{E}$, the objects of $\mathrm{P}(1)^{s}$ are precisely the structure sheaves $\boldsymbol{k}(x)$ of closed points $x \in \boldsymbol{E}$. In order to understand the structure of all the other categories $\mathrm{P}(\varphi)^{s}$, we use Fourier-Mukai transforms. Our main technical tool will be the transform $\mathbb{F}$ which was studied in [BK05]. It depends on the choice of a regular point $p_{0} \in \boldsymbol{E}$. Let us briefly recall its definition and main properties. It was defined with the aid of Seidel-Thomas twists [ST01], which are functors $T_{E}: \mathrm{D}_{\text {coh }}^{\mathrm{b}}(\boldsymbol{E}) \rightarrow \mathrm{D}_{\text {coh }}^{\mathrm{b}}(\boldsymbol{E})$ depending on a spherical object $E \in \mathrm{D}_{\text {coh }}^{\mathrm{b}}(\boldsymbol{E})$. On objects, these functors are characterised by the existence of a distinguished triangle

$$
\boldsymbol{R} \operatorname{Hom}(E, F) \otimes E \longrightarrow F \longrightarrow T_{E}(F) \stackrel{+}{\longrightarrow} .
$$

If $p_{0} \in \boldsymbol{E}$ is a smooth point, the functor $T_{\boldsymbol{k}\left(p_{0}\right)}$ is isomorphic to the tensor product with the locally free sheaf $\mathcal{O}_{\boldsymbol{E}}\left(p_{0}\right)$; see [ST01, 3.11]. We defined

$$
\mathbb{F}:=T_{\boldsymbol{k}\left(p_{0}\right)} T_{\mathcal{O}} T_{\boldsymbol{k}\left(p_{0}\right)}
$$

In [ST01] it was shown that twist functors can be described as integral transforms and that $\mathbb{F}$ is isomorphic to the functor $\mathrm{FM}^{\mathcal{P}}$, which is given by

$$
\operatorname{FM}^{\mathcal{P}}(\cdot):=\boldsymbol{R} \pi_{2 *}\left(\mathcal{P} \stackrel{\boldsymbol{L}}{\otimes} \pi_{1}^{*}(\cdot)\right),
$$

where $\mathcal{P}=\mathcal{I}_{\Delta} \otimes \pi_{1}^{*} \mathcal{O}\left(p_{0}\right) \otimes \pi_{2}^{*} \mathcal{O}\left(p_{0}\right)[1]$. This is a shift of a coherent sheaf on $\boldsymbol{E} \times \boldsymbol{E}$, on which we denote the ideal of the diagonal by $\mathcal{I}_{\Delta} \subset \mathcal{O}_{\boldsymbol{E} \times \boldsymbol{E}}$ and the two projections by $\pi_{1}, \pi_{2}$.

In order to understand the effect of $\mathbb{F}$ on rank and degree, we look at the distinguished triangle

$$
\boldsymbol{R} \operatorname{Hom}(\mathcal{O}, F) \otimes \mathcal{O} \longrightarrow F \longrightarrow T_{\mathcal{O}}(F) \stackrel{+}{\longrightarrow} .
$$

The additivity of rank and degree implies $\operatorname{rk}\left(T_{\mathcal{O}}(F)\right)=\operatorname{rk}(F)-\operatorname{deg}(F)$ and $\operatorname{deg}\left(T_{\mathcal{O}}(F)\right)=\operatorname{deg}(F)$. On the other hand, it is well known that $\operatorname{deg}\left(T_{\boldsymbol{k}\left(p_{0}\right)}(F)\right)=\operatorname{deg}(F)+\operatorname{rk}(F)$ and $\operatorname{rk}\left(T_{\boldsymbol{k}\left(p_{0}\right)}(F)\right)=\operatorname{rk}(F)$. So, if we use $\left[\mathcal{O}_{\boldsymbol{E}}\right],-\left[\boldsymbol{k}\left(p_{0}\right)\right]$ as a basis of $\mathrm{K}(\boldsymbol{E})$, which means that we use coordinates (rk, $-\mathrm{deg}$ ), then the action of $T_{\mathcal{O}}, T_{\boldsymbol{k}\left(p_{0}\right)}$ and $\mathbb{F}$ on $\mathrm{K}(\boldsymbol{E})$ is given respectively by the matrices

$$
\left(\begin{array}{ll}
1 & 1 \\
0 & 1
\end{array}\right), \quad\left(\begin{array}{cc}
1 & 0 \\
-1 & 1
\end{array}\right) \text { and } \quad\left(\begin{array}{cc}
0 & 1 \\
-1 & 0
\end{array}\right)
$$




\section{Burban And B. Kreußler}

In particular, for any object $F \in \mathrm{D}_{\text {coh }}^{\mathrm{b}}(\boldsymbol{E})$ which has a slope, we have $\mu\left(T_{\boldsymbol{k}\left(p_{0}\right)}(F)\right)=\mu(F)+1$ and $\mu(\mathbb{F}(F))=-1 / \mu(F)$ using the usual conventions in dealing with $\infty$.

If $F$ is a sheaf or a twist thereof, we defined the phase $\varphi(F)$. In order to understand the effect of $\mathbb{F}$ on phases, it is not sufficient to know its effect on the slope. This is because the slope determines the phase modulo $2 \mathbb{Z}$ only. However, if $F$ is a coherent sheaf, the description of $\mathbb{F}$ as $\mathrm{FM}^{\mathcal{P}}$ shows that $\mathbb{F}(F)$ can have non-vanishing cohomology in degrees -1 and 0 only. If, in addition, $\mathbb{F}(F)$ is a shifted sheaf, this implies that $\varphi(\mathbb{F}(F)) \in(0,2]$. From the formula for the slope it is now clear that $\varphi(\mathbb{F}(F))=\varphi(F)+\frac{1}{2}$ for any shifted coherent sheaf $F$.

The following result was first shown in [BBHM02]. We give an independent proof here, which was inspired by [BBHM02, Lemma 3.1].

Theorem 4.1. The functor $\mathbb{F}$ sends semi-stable sheaves to semi-stable sheaves.

Proof. Note that, by definition, a semi-stable sheaf of positive rank is automatically torsion free. The only sheaf with degree and rank equal to zero is the zero sheaf. Throughout this proof, we let $\mathcal{F}$ be a semi-stable sheaf on $\boldsymbol{E}$. If $\operatorname{deg}(\mathcal{F})=0$ this sheaf is torsion free and the claim was shown in [BK05, Theorem 2.21]; see also [FM03]. For the sake of clarity we would like to stress here the fact that $[$ BK05, §2] deals with nodal as well as cuspidal Weierstraß curves.

Next, suppose that $\operatorname{deg}(\mathcal{F})>0$. If $\operatorname{rk}(\mathcal{F})=0, \mathcal{F}$ is a coherent torsion sheaf. Again, the claim follows from [BK05, Theorems 2.21 and 2.18], where it was shown that $\mathbb{F} \circ \mathbb{F}=i^{*}[1]$, for any Weierstraß curve. Here, $i: \boldsymbol{E} \rightarrow \boldsymbol{E}$ is the involution which fixes the singularity and which corresponds to taking the inverse on the smooth part of $\boldsymbol{E}$ with its group structure in which $p_{0}$ is the neutral element.

Therefore, we may suppose $\mathcal{F}$ is torsion free. As observed before, the complex $\mathbb{F}(\mathcal{F}) \in \mathrm{D}_{\text {coh }}^{\mathrm{b}}(\boldsymbol{E})$ can have non-vanishing cohomology in degrees -1 and 0 only. We are going to show that $\mathbb{F}(\mathcal{F})[-1]$ is a sheaf, which is equivalent to the vanishing of the cohomology object $\mathcal{H}^{0}(\mathbb{F}(\mathcal{F})) \in \operatorname{Coh}_{E}$. Recall from [BK05, Lemma 2.13], that for any smooth point $x \in \boldsymbol{E}$ the sheaf of degree zero $\mathcal{O}\left(x-p_{0}\right)$ satisfies $\mathbb{F}\left(\mathcal{O}\left(x-p_{0}\right)\right) \cong T_{\mathcal{O}}(\mathcal{O}(x)) \cong \boldsymbol{k}(x)$. Moreover, if $s \in \boldsymbol{E}$ denotes the singular point, $n: \mathbb{P}^{1} \rightarrow \boldsymbol{E}$ the normalisation and $\widetilde{\mathcal{O}}:=n_{*}\left(\mathcal{O}_{\mathbb{P}^{1}}\right)$, then $\mathbb{F}\left(\widetilde{\mathcal{O}}\left(-p_{0}\right)\right) \cong T_{\mathcal{O}}(\widetilde{\mathcal{O}}) \cong \boldsymbol{k}(s)$. The sheaf $\widetilde{\mathcal{O}}\left(-p_{0}\right)$ has degree zero on $\boldsymbol{E}$. Because $\mathbb{F}$ is an equivalence, we obtain isomorphisms

$$
\operatorname{Hom}(\mathbb{F}(\mathcal{F}), \boldsymbol{k}(x)) \cong \operatorname{Hom}\left(\mathcal{F}, \mathcal{O}\left(x-p_{0}\right)\right)
$$

and

$$
\operatorname{Hom}(\mathbb{F}(\mathcal{F}), \boldsymbol{k}(s)) \cong \operatorname{Hom}\left(\mathcal{F}, \widetilde{\mathcal{O}}\left(-p_{0}\right)\right),
$$

where $x \in \boldsymbol{E}$ is an arbitrary smooth point. These vector spaces vanish as $\mathcal{F}$ was assumed to be semi-stable and of positive degree.

Because cohomology of the complex $\mathbb{F}(\mathcal{F})$ vanishes in positive degree, there is a canonical morphism $\mathbb{F}(\mathcal{F}) \rightarrow \mathcal{H}^{0}(\mathbb{F}(\mathcal{F}))$ in $\mathrm{D}_{\text {coh }}^{\mathrm{b}}(\boldsymbol{E})$, which induces an injection of functors $\operatorname{Hom}\left(\mathcal{H}^{0}(\mathbb{F}(\mathcal{F})), \cdot\right) \hookrightarrow$ $\operatorname{Hom}(\mathbb{F}(\mathcal{F}), \cdot)$. Therefore, the vanishing which was obtained above shows that

$$
\operatorname{Hom}\left(\mathcal{H}^{0}(\mathbb{F}(\mathcal{F})), \boldsymbol{k}(y)\right)=0
$$

for any point $y \in \boldsymbol{E}$. This implies the vanishing of the sheaf $\mathcal{H}^{0}(\mathbb{F}(\mathcal{F}))$. Hence, $\widehat{\mathcal{F}}:=\mathbb{F}(\mathcal{F})[-1]$ is a coherent sheaf and the definition of $\mathbb{F}$ implies that there is an exact sequence of coherent sheaves

$$
0 \longrightarrow \widehat{\mathcal{F}}\left(-p_{0}\right) \longrightarrow H^{0}\left(\mathcal{F}\left(p_{0}\right)\right) \otimes \mathcal{O}_{\boldsymbol{E}} \longrightarrow \mathcal{F}\left(p_{0}\right) \longrightarrow 0 .
$$

This sequence implies, in particular, that $\widehat{\mathcal{F}}$ is torsion free.

Before we proceed to show that $\widehat{\mathcal{F}}$ is semi-stable, we apply duality to prove that $\mathbb{F}(\mathcal{F})$ is a sheaf if $\operatorname{deg}(\mathcal{F})<0$. Let us denote the dualising functor by $\mathbb{D}:=\boldsymbol{R} \mathcal{H o m}\left(\cdot, \mathcal{O}_{\boldsymbol{E}}\right)$. This functor 


\section{DERIVED CATEGORIES OF IRREDUCIBLE PROJECTIVE CURVES}

satisfies $\mathbb{D D} \cong \mathbf{1}$. In [BK04, Corollary 3.4], we have shown that there exists an isomorphism

$$
\mathbb{D F}[-1] \cong i^{*} \mathbb{F D} \text {. }
$$

Using $\mathbb{D} \circ[1] \cong[-1] \circ \mathbb{D}$, this implies that

$$
\mathbb{F} \cong \mathbb{D} i^{*}[-1] \mathbb{F D} .
$$

Because $\mathcal{F}$ is a torsion free sheaf on a curve, it is Cohen-Macaulay and, since $\boldsymbol{E}$ is Gorenstein, this implies $\mathcal{E} x t^{i}(\mathcal{F}, \mathcal{O})=0$ for any $i>0$. Therefore, we have $\mathbb{D}(\mathcal{F}) \cong \mathcal{F}^{\vee}$ and this is a semi-stable coherent sheaf of positive degree. Thus, $[-1] \circ \mathbb{F}$ sends $\mathcal{F}^{\vee}$ to a torsion free sheaf, on which $\mathbb{D}$ is just the usual dual. Now, we see that $\mathbb{F}(\mathcal{F})$ is a torsion free sheaf if $\mathcal{F}$ was semi-stable and of negative degree.

It remains to prove that $\mathbb{F}$ preserves semi-stability. If $\operatorname{deg}(\mathcal{F})=0$ or $\mathcal{F}$ is a torsion sheaf, this was shown for any Weierstraß curve in [BK05]. If $\operatorname{deg}(\mathcal{F}) \neq 0$ the proof is based upon $\mathbb{F F}[-1] \cong i^{*}$; see $\left[\right.$ BK05, Theorem 2.18]. Suppose $\operatorname{deg}(\mathcal{F})>0$, then $\mathbb{F}(\widehat{\mathcal{F}}) \cong i^{*}(\mathcal{F})$ and this is a coherent sheaf. If $\widehat{\mathcal{F}}$ were not semi-stable, there would exist a semi-stable sheaf $\mathcal{G}$ with $\mu(\widehat{\mathcal{F}})>\mu(\mathcal{G})$ and a non-zero morphism $\widehat{\mathcal{F}} \rightarrow \mathcal{G}$. Because $\mu(\widehat{\mathcal{F}})=-1 / \mu(\mathcal{F})<0, \mathbb{F}(\mathcal{G})$ is a coherent sheaf and application of $\mathbb{F}$ produces a non-zero morphism $i^{*}(\mathcal{F}) \cong \mathbb{F}(\widehat{\mathcal{F}}) \rightarrow \mathbb{F}(\mathcal{G})$. However, $\mu\left(i^{*}(\mathcal{F})\right)=\mu(\mathcal{F})>-1 / \mu(\mathcal{G})=$ $\mu(\mathbb{F}(\mathcal{G}))$ contradicts semi-stability of $i^{*}(\mathcal{F})$. Hence, $\widehat{\mathcal{F}}$ is semi-stable. The proof in the case $\operatorname{deg}(\mathcal{F})<$ 0 starts with a non-zero morphism $\mathcal{U} \rightarrow \mathbb{F}(\mathcal{F})$ and proceeds similarly.

It was shown in $[\mathrm{BK} 05]$ that we obtain an action of the group $\widetilde{\mathrm{SL}}(2, \mathbb{Z})$ on $\mathrm{D}_{\text {coh }}^{\mathrm{b}}(\boldsymbol{E})$ by sending generators of this group to $T_{\mathcal{O}}, T_{\boldsymbol{k}\left(p_{0}\right)}$ and the translation functor [1] respectively. Let us denote

$$
\mathrm{Q}:=\{\varphi \in \mathbb{R} \mid \mathrm{P}(\varphi) \text { contains a non-zero object }\} .
$$

The action of a group $G$ on $\mathrm{Q}$ is called monotone if $\varphi \leqslant \psi$ implies $g \cdot \varphi \leqslant g \cdot \psi$ for every $g \in G$ and $\varphi, \psi \in \mathrm{Q}$.

Proposition 4.2. The $\widetilde{\mathrm{SL}}(2, \mathbb{Z})$-action on $\mathrm{D}_{\text {coh }}^{\mathrm{b}}(\boldsymbol{E})$ induces a monotone and transitive action on the set $\mathrm{Q}$. All isotropy groups of this action are isomorphic to $\mathbb{Z}$.

Proof. As seen above, for any $\psi \in \mathrm{Q}$ and $0 \neq A \in \mathrm{P}(\psi)$, we have $\varphi(\mathbb{F}(A))=\varphi(A)+\frac{1}{2}$ and $\mu\left(T_{\boldsymbol{k}\left(p_{0}\right)}(A)\right)=\mu(A)+1$. Therefore, by Theorem 4.1 it is clear that we obtain an induced monotone action of $\widetilde{\mathrm{SL}}(2, \mathbb{Z})$ on $\mathrm{Q}$. The group $\mathrm{SL}(2, \mathbb{Z})$ acts transitively on the set of all pairs of co-prime integers which we interpret as primitive vectors of the lattice $\mathbb{Z} \oplus i \mathbb{Z} \subset \mathbb{C}$. Hence, the action of $\widetilde{\mathrm{SL}}(2, \mathbb{Z})$ on $\mathrm{Q}$ is transitive as well. So, all isotropy groups are isomorphic. Finally, it is easy to see that the isotropy group of $1 \in \mathrm{Q}$ is generated by $T_{\boldsymbol{k}\left(p_{0}\right)}$.

As an important consequence we obtain the following clear structure result for the slices $\mathrm{P}(\varphi)$.

Corollary 4.3. The category $\mathrm{P}(\varphi)$ of semi-stable objects of phase $\varphi \in \mathrm{Q}$ is equivalent to the category $\mathrm{P}(1)$ of torsion sheaves. Any such equivalence restricts to an equivalence between $\mathrm{P}(\varphi)^{s}$ and $\mathrm{P}(1)^{s}$. Under such an equivalence, stable vector bundles correspond to structure sheaves of smooth points. Moreover, if $\varphi \in(0,1) \cap \mathrm{Q}, \mathrm{P}(\varphi)^{s}$ contains a unique torsion free sheaf, which is not locally free. It corresponds to the structure sheaf $\boldsymbol{k}(s) \in \mathrm{P}(1)^{s}$ of the singular point.

Recall that an object $E \in \mathrm{D}_{\text {coh }}^{\mathrm{b}}(\boldsymbol{E})$ is called perfect if it is isomorphic in the derived category to a bounded complex of locally free sheaves of finite rank. Thus, a sheaf or shift thereof is called perfect if it is perfect as an object in $\mathrm{D}_{\text {coh }}^{\mathrm{b}}(\boldsymbol{E})$. If $\boldsymbol{E}$ is smooth, any object in $\mathrm{D}_{\text {coh }}^{\mathrm{b}}(\boldsymbol{E})$ is perfect. However, if $s \in \boldsymbol{E}$ is a singular point, the torsion sheaf $\boldsymbol{k}(s)$ is not perfect.

If $\boldsymbol{E}$ is singular with one singularity $s \in \boldsymbol{E}$, the category $\mathrm{P}(1)^{s}$ contains precisely one object which is not perfect, the object $\boldsymbol{k}(s)$. Hence, by Proposition 4.2 , for any $\varphi \in Q$ there is precisely 


\section{Burban and B. KreußleR}

one element in $\mathrm{P}(\varphi)^{s}$ which is not perfect. We shall refer to it as the extreme stable element with phase $\varphi$. So, the sheaf $\boldsymbol{k}(s)$ is the extreme stable element with phase 1 . The extreme stable element is never locally free. A stable object is either perfect or extreme.

We shall need the following version of Serre duality, which can be deduced easily from standard versions.

If $E, F \in \mathrm{D}_{\text {coh }}^{\mathrm{b}}(\boldsymbol{E})$ and at least one of them is perfect, then there is a bi-functorial isomorphism

$$
\operatorname{Hom}(E, F) \cong \operatorname{Hom}(F, E[1])^{*} \text {. }
$$

If neither of the objects is perfect, this is no longer true. For example, $\operatorname{Hom}(\boldsymbol{k}(s), \boldsymbol{k}(s)) \cong \boldsymbol{k}$, but $\operatorname{Hom}(\boldsymbol{k}(s), \boldsymbol{k}(s)[1]) \cong \operatorname{Ext}^{1}(\boldsymbol{k}(s), \boldsymbol{k}(s)) \cong \boldsymbol{k}^{2}$.

Any object $X$ in the Abelian category $\mathrm{P}(\varphi)$ has a Jordan-Hölder filtration (JHF)

$$
0 \subset F_{n} X \subset \cdots \subset F_{1} X \subset F_{0} X=X
$$

with stable JH-factors $J_{i}=F_{i} X / F_{i+1} X \in \mathrm{P}(\varphi)^{s}$. The graded object $\bigoplus_{i=0}^{n} J_{i}$ is determined by $X$. Observe that for any two objects $J \neq J^{\prime} \in \mathrm{P}(\varphi)^{s}$ we can apply Serre duality because at most one of them is non-perfect.

Corollary 4.4.

(i) If $\varphi, \psi \in \mathrm{Q}$ with $\varphi-1<\psi \leqslant \varphi$ there exists $\Phi \in \widetilde{\mathrm{SL}}(2, \mathbb{Z})$ such that $\Phi(\varphi)=1$ and $\Phi(\psi) \in(0,1]$.

(ii) If $A, B \in \mathrm{P}(\varphi)^{s}$, then $A \cong B \Longleftrightarrow \operatorname{Hom}(A, B) \neq 0$.

(iii) If $0 \neq X \in \mathrm{P}(\varphi)$ and $0 \neq Y \in \mathrm{P}(\psi)$ with $\varphi<\psi<\varphi+1$, then $\operatorname{Hom}(X, Y) \neq 0$.

(iv) If $J \in \mathrm{P}(\varphi)^{s}$ is not a $J H$-factor of $X \in \mathrm{P}(\varphi)$, for all $i \in \mathbb{Z}$ we have $\operatorname{Hom}(J, X[i])=0$.

(v) If $X \in \mathrm{P}(\varphi)$ is indecomposable, all its JH-factors are isomorphic to each other.

(vi) If $X, Y \in \mathrm{P}(\varphi)$ are non-zero indecomposable objects, both with the same JH-factor, then $\operatorname{Hom}(X, Y) \neq 0$.

Proof. (i) This follows from Proposition 4.2 because the shift functor corresponds to an element in the centre of $\widetilde{\mathrm{SL}}(2, \mathbb{Z})$ and therefore $\Phi(\mathrm{P}(\varphi))=\mathrm{P}(1)$ implies $\Phi(\mathrm{P}(\varphi-1))=\mathrm{P}(0)$.

(ii) The statement is clear in the case $\varphi=1$ and follows from (i) in the general case.

(iii) Using (i) we can assume $\psi=1$, which means that $Y$ is a coherent torsion sheaf. By Proposition 4.2 this implies $\varphi \in(0,1)$ and $X$ is a torsion free coherent sheaf. If $Y \in \mathrm{P}(1)^{s}$ the statement is clear, because any torsion free sheaf has a non-zero morphism to any $Y=\boldsymbol{k}(x), x \in \boldsymbol{E}$. If $Y \in \mathrm{P}(1)$ is arbitrary, there exists a point $x \in \boldsymbol{E}$ and a non-zero morphism $\boldsymbol{k}(x) \rightarrow Y$. The claim follows now from left-exactness of the functor $\operatorname{Hom}(X, \cdot)$.

(iv) If $J^{\prime} \in \mathrm{P}(\varphi)^{s}$ is a JH-factor of $X$, we have $J \not J^{\prime}$. From (ii) and Serre duality together with Lemma 3.9 we obtain $\operatorname{Hom}\left(J, J^{\prime}[i]\right)=0$ for any $i \in \mathbb{Z}$. Using the JHF of $X$, the claim now follows.

(v) It is easy to prove by induction that any $X \in \mathrm{P}(\varphi)$ can be split as a finite direct sum $X \cong \bigoplus X_{k}$, where each $X_{k}$ has all JH-factors isomorphic to a single element $J_{k} \in \mathrm{P}(\varphi)^{s}$. This implies the claim.

(vi) By (i) we may assume $\varphi(X)=\varphi(Y)=1$. This means that both objects are indecomposable torsion sheaves with support at the same point $x \in \boldsymbol{E}$. Such sheaves always have an epimorphism to and a monomorphism from the object $\boldsymbol{k}(x)$, hence the claim.

It is interesting and important to note that an indecomposable semi-stable object can be perfect even though all its JH-factors are extreme. This is made explicit in [BK05, §4], in the case of the category $\mathrm{P}(1)$ of coherent torsion sheaves. If $\boldsymbol{E}$ is nodal, there are two kinds of indecomposable torsion sheaves with support at the node $s \in \boldsymbol{E}$ : the so-called bands and strings. The bands are 


\section{DERIVED CATEGORIES OF IRREDUCIBLE PROJECTIVE CURVES}

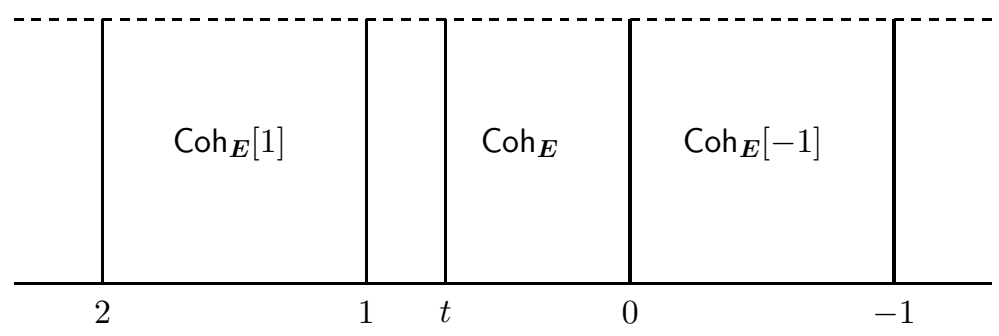

Figure 1. Slices.

perfect, whereas the strings are not perfect. Using the action of $\widetilde{\mathrm{SL}}(2, \mathbb{Z})$ this carries over to all other categories $\mathrm{P}(\varphi)$ with $\varphi \in \mathrm{Q}$.

An object $X \in \mathrm{P}(\varphi)$ will be called extreme if it does not have a direct summand which is perfect. This implies that, but is not equivalent to, the property that all its JH-factors are extreme. An example can be found below (see Example 4.9). From the above we deduce that any $X \in \mathrm{P}(\varphi)$ can be split as a direct sum $X \cong X^{e} \oplus X^{p}$ with $X^{e}$ extreme and $X^{p}$ perfect. All direct summands of the extreme part have the unique extreme stable element with phase $\varphi$ as its JH-factors. On the other hand, all the direct summands of $X^{p}$ are perfect and they can have any object of $\mathrm{P}(\varphi)^{s}$ as JH-factor.

Corollary 4.5. Any coherent sheaf $\mathcal{F}$ with $\operatorname{End}(\mathcal{F})=\boldsymbol{k}$ is stable.

Proof. The assumption implies that $\mathcal{F}$ is indecomposable. If $\mathcal{F}$ were not even semi-stable, it would have at least two HN-factors. Using Corollary 4.4, we may assume that $\varphi_{+}(\mathcal{F})=1$. Thus, $\mathcal{F}$ is a coherent sheaf which is neither torsion nor torsion free. This implies that there is a noninvertible endomorphism $\mathcal{F} \rightarrow \boldsymbol{k}(x) \rightarrow \operatorname{tors}(\mathcal{F}) \rightarrow \mathcal{F}$, in contradiction to the assumption. Hence, $\mathcal{F} \in \mathrm{P}(\varphi)$ is semi-stable. Let $\mathcal{J} \in \mathrm{P}(\varphi)$ be its JH-factor. From Corollary 4.4(vi) we obtain a non-zero endomorphism $\mathcal{F} \rightarrow \mathcal{J} \rightarrow \mathcal{F}$, which can only be an isomorphism if $\mathcal{F} \cong \mathcal{J}$, so $\mathcal{F}$ is indeed stable.

The following method can be used to visualise the structure of the category $D_{\text {coh }}^{\mathrm{b}}(\boldsymbol{E})$ : the vertical slices in Figure 1 are thought to correspond to the categories $\mathrm{P}(t)^{s}$ of stable objects. They are nonempty if and only if $t \in \mathrm{Q}$, i.e. $\mathbb{R} \exp (\pi i t) \cap \mathbb{Z}^{2} \neq\{(0,0)\}$. A point on such a slice represents a stable object. The extreme stable objects are those which lie on the dashed upper horizontal line. The labelling below the picture reflects the phases of the slices. We have chosen to let it decrease from left to right in order to have objects with cohomology in negative degrees on the left and with positive degrees on the right.

By Proposition 4.2 , the group $\widetilde{\mathrm{SL}}(2, \mathbb{Z})$ acts on the set of all stable objects, hence it acts on such pictures. This action sends slices to slices and acts transitively on the set of slices with phase $t \in \mathrm{Q}$. The dashed line of extreme stable objects is invariant under this action.

Any indecomposable object $0 \neq X \in \mathrm{D}_{\text {coh }}^{\mathrm{b}}(\boldsymbol{E})$ has a shadow in such a picture: it is the set of all stable objects which occur as JH-factors in the HN-factors of $X$. If this set consists of more than one point, the shadow is obtained by connecting these points by line segments.

The following proposition shows that the shadow of an indecomposable object which consists of more than one point is completely contained in the extreme line.

Figure 2 shows the shadows of five different indecomposable objects:

(a) $X_{1} \in \mathrm{Coh}_{\boldsymbol{E}}$ an indecomposable torsion sheaf;

(b) $X_{2} \in \operatorname{Coh}_{\boldsymbol{E}}[-1]$ the shift of an indecomposable semi-stable locally free sheaf; 
I. Burban AND B. Kreußler

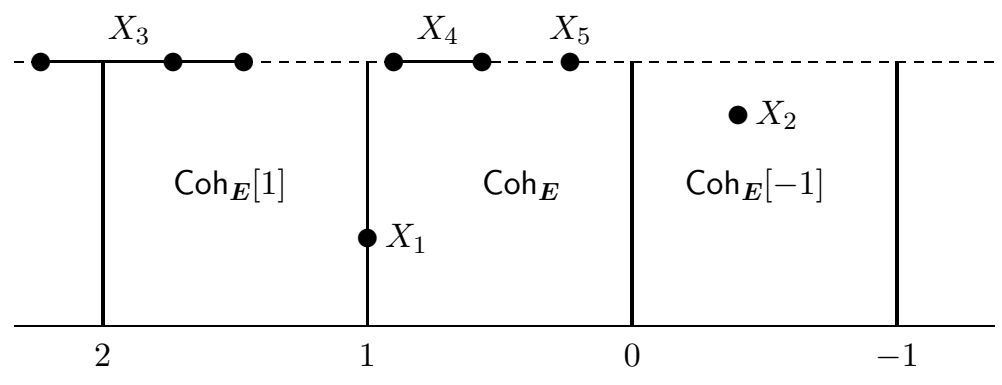

FIGURE 2. Shadows.

(c) $X_{3}$ a genuine complex with three extreme HN-factors, one in $\mathrm{Coh}_{\boldsymbol{E}}[2]$ and the other two in $\mathrm{Coh}_{E}[1]$

(d) $X_{4}$ an indecomposable torsion free sheaf which is not semi-stable;

(e) $X_{5} \in \operatorname{Coh}_{E}$ an indecomposable and semi-stable torsion free sheaf which could be perfect or not (a band or a string in the language of representation theory).

The shadow of an indecomposable object is a single point if and only if this object is semi-stable.

Proposition 4.6. Let $X \in \mathrm{D}_{\text {coh }}^{\mathrm{b}}(\boldsymbol{E})$ be an indecomposable object which is not semi-stable. Then all $\mathrm{HN}$-factors of $X$ are extreme.

Proof. Let

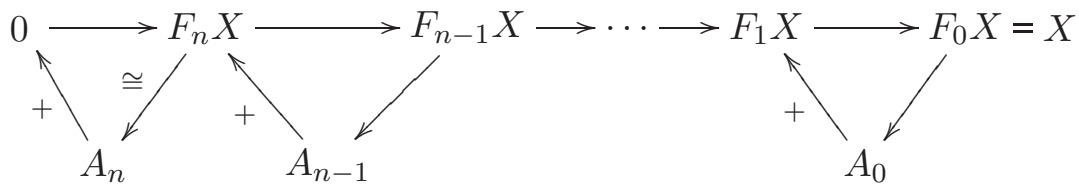

be an HNF of $X$. If the HN-factor $A_{i}$ were not extreme, it could be split into a direct sum $A_{i} \cong$ $A_{i}^{\prime} \oplus A_{i}^{\prime \prime}$ with $0 \neq A_{i}^{\prime}$ perfect and $A_{i}^{\prime}, A_{i}^{\prime \prime} \in \mathrm{P}\left(\varphi_{i}\right)$. Because $\varphi_{-}\left(F_{i+1} X\right)>\varphi_{i}=\varphi\left(A_{i}^{\prime}\right)$, Lemma 3.9 and Serre duality imply

$$
\operatorname{Hom}\left(A_{i}^{\prime}, F_{i+1} X[1]\right) \cong \operatorname{Hom}\left(F_{i+1} X, A_{i}^{\prime}\right)^{*}=0 .
$$

Hence, we can apply Lemma 3.11 to the distinguished triangle

$$
F_{i+1} X \longrightarrow F_{i} X \longrightarrow A_{i} \stackrel{+}{\longrightarrow}
$$

and obtain a decomposition $F_{i} X \cong F_{i}^{\prime} X \oplus A_{i}^{\prime}$. We proceed by descending induction on $j \leqslant i$ to show that there exist decompositions $F_{j} X \cong F_{j}^{\prime} X \oplus A_{i}^{\prime}$. This is obtained from Lemma 3.11 applied to the distinguished triangle

$$
F_{j}^{\prime} X \oplus A_{i}^{\prime} \longrightarrow F_{j-1} X \longrightarrow A_{j-1} \stackrel{+}{\longrightarrow}
$$

and using Lemma 3.9, Serre duality and $\varphi\left(A_{i}^{\prime}\right)>\varphi\left(A_{j-1}\right)$ to get

$$
\operatorname{Hom}\left(A_{j-1}, A_{i}^{\prime}[1]\right) \cong \operatorname{Hom}\left(A_{i}^{\prime}, A_{j-1}\right)^{*}=0 .
$$

We obtain a decomposition $X=F_{0} X \cong F_{0}^{\prime} X \oplus A_{i}^{\prime}$ in which we have $A_{i}^{\prime} \neq 0$. Because $X$ was assumed to be indecomposable, we should have $X \cong A_{i}^{\prime}$, but this was excluded by assumption. This contradiction shows that all $\mathrm{HN}$-factors $A_{i}$ are necessarily extreme.

Corollary 4.7. There exist four types of indecomposable objects in the category $\mathrm{Coh}_{\boldsymbol{E}}$ :

(i) semi-stable with perfect $\mathrm{JH}$-factor;

(ii) semi-stable, perfect but its JH-factor extreme; 


\section{DERIVED CATEGORIES OF IRREDUCIBLE PROJECTIVE CURVES}

(iii) semi-stable and extreme;

(iv) not semi-stable, with all its $H N$-factors extreme.

A similar statement is true for $D_{\text {coh }}^{\mathrm{b}}(\boldsymbol{E})$. In this case, the objects of types (i), (ii) and (iii) are shifts of coherent sheaves, whereas genuine complexes are possible for objects of type (iv). Types (ii), (iii) and (iv) were not available in the smooth case.

Examples of type (i) are simple vector bundles and structure sheaves $\boldsymbol{k}(x)$ of smooth points $x \in \boldsymbol{E}$. All indecomposable objects with a shadow not on the extreme line fall into type (i). Under the equivalences of Corollary 4.3, indecomposable semi-stable locally free sheaves with extreme JHfactor correspond, in the nodal case, precisely to those torsion sheaves with support at the node $s$, which are called bands (see [BK05]). Examples of type (iii) are the stable coherent sheaves which are not locally free and the structure sheaf $\boldsymbol{k}(s)$ of the singular point $s \in \boldsymbol{E}$. Moreover, in the nodal case, the torsion sheaves with support at $s$, which are called strings in [BK05], are of type (iii) as well. Examples of objects of type (iv) are given below.

Example 4.8. We shall construct torsion free sheaves on nodal $\boldsymbol{E}$ with an arbitrary finite number of HN-factors. This implies that the number of points in a shadow of an indecomposable object in $\mathrm{D}_{\text {coh }}^{\mathrm{b}}(\boldsymbol{E})$ is not bounded.

Recall from [DG01] that any indecomposable torsion free sheaf which is not locally free is isomorphic to a sheaf $\mathcal{S}(\boldsymbol{d})=p_{n *} \mathcal{L}(\boldsymbol{d})$. We use here the notation of $[\mathrm{BK} 05, \S 3.5]$, so that $p_{n}: \boldsymbol{I}_{\boldsymbol{n}} \rightarrow \boldsymbol{E}$ denotes a certain morphism from the chain $\boldsymbol{I}_{\boldsymbol{n}}$ of $n$ smooth rational curves to the nodal curve $\boldsymbol{E}$. If $\boldsymbol{d}=\left(d_{1}, \ldots, d_{n}\right) \in \mathbb{Z}^{n}$, we denote by $\mathcal{L}(\boldsymbol{d})$ the line bundle on $\boldsymbol{I}_{\boldsymbol{n}}$ which has degree $d_{\nu}$ on the $\nu$ th component of $\boldsymbol{I}_{\boldsymbol{n}}$. We know $\operatorname{rk}(\mathcal{S}(\boldsymbol{d}))=n$ and $\operatorname{deg}(\mathcal{S}(\boldsymbol{d}))=1+\sum d_{\nu}$. We obtain, in particular, that for any $\varphi \in \mathrm{Q} \cap(0,1)$ there exist $n \in \mathbb{Z}$ and $\boldsymbol{d}(\varphi) \in \mathbb{Z}^{n}$ such that $\mathcal{S}(\boldsymbol{d}(\varphi))$ is the unique extreme element in $\mathrm{P}(\varphi)^{s}$. On the other hand, if $\boldsymbol{d}^{\prime} \in \mathbb{Z}^{n^{\prime}}, \boldsymbol{d}^{\prime \prime} \in \mathbb{Z}^{n^{\prime \prime}}$ and $\boldsymbol{d}=\left(\boldsymbol{d}_{+}^{\prime}, \boldsymbol{d}^{\prime \prime}\right) \in \mathbb{Z}^{n^{\prime}+n^{\prime \prime}}$, where $\boldsymbol{d}_{+}^{\prime}$ is obtained from $\boldsymbol{d}^{\prime}$ by adding 1 to the last component, we have an exact sequence

$$
0 \longrightarrow \mathcal{S}\left(\boldsymbol{d}^{\prime}\right) \longrightarrow \mathcal{S}(\boldsymbol{d}) \longrightarrow \mathcal{S}\left(\boldsymbol{d}^{\prime \prime}\right) \longrightarrow 0
$$

(see for example [Moz04]). Hence, if we start with a sequence $0<\varphi_{0}<\varphi_{1}<\cdots<\varphi_{m}<1$ where $\varphi_{\nu} \in \mathrm{Q}$ and define

$$
\boldsymbol{d}^{(m)}=\boldsymbol{d}\left(\varphi_{m}\right) \quad \text { and } \quad \boldsymbol{d}^{(\nu)}=\left(\boldsymbol{d}_{+}^{(\nu+1)}, \boldsymbol{d}\left(\varphi_{\nu}\right)\right) \text { for } m>\nu \geqslant 0,
$$

we obtain an indecomposable torsion free sheaf $\mathcal{S}\left(\boldsymbol{d}^{(0)}\right)$ whose HN-factors are the extreme stable sheaves $\mathcal{S}\left(\boldsymbol{d}\left(\varphi_{\nu}\right)\right) \in \mathrm{P}\left(\varphi_{\nu}\right), 0 \leqslant \nu \leqslant m$. The HNF of this sheaf is given by

$$
\mathcal{S}\left(\boldsymbol{d}^{(m)}\right) \subset \mathcal{S}\left(\boldsymbol{d}^{(m-1)}\right) \subset \cdots \subset \mathcal{S}\left(\boldsymbol{d}^{(0)}\right) .
$$

The sheaf $\mathcal{S}\left(\boldsymbol{d}^{(0)}\right)$ is of type (iv) and not perfect.

Example 4.9. Suppose $\boldsymbol{E}$ is nodal and let $\pi: C_{2} \rightarrow \boldsymbol{E}$ be an étale morphism of degree two, where $C_{2}$ denotes a reducible curve which has two components, both isomorphic to $\mathbb{P}^{1}$ and which intersect transversally at two distinct points. By $i_{\nu}: \mathbb{P}^{1} \rightarrow \boldsymbol{E}, \nu=1,2$, we denote the morphisms which are induced by the embeddings of the two components of $C_{2}$. There is a $\boldsymbol{k}^{\times}$-family of line bundles on $C_{2}$ whose restriction to one component is $\mathcal{O}_{\mathbb{P}^{1}}(-2)$ and to the other is $\mathcal{O}_{\mathbb{P}^{1}}(2)$. The element in $\boldsymbol{k}^{\times}$ corresponds to a gluing parameter over one of the two singularities of $C_{2}$. If $\mathcal{L}$ denotes one such line bundle, $\mathcal{E}:=\pi_{*} \mathcal{L}$ is an indecomposable vector bundle of rank two and degree zero on $\boldsymbol{E}$. Let us fix notation so that $i_{1}^{*} \mathcal{E} \cong \mathcal{O}_{\mathbb{P}^{1}}(-2)$ and $i_{2}^{*} \mathcal{E} \cong \mathcal{O}_{\mathbb{P}^{1}}(2)$. There is an exact sequence of coherent sheaves on $\boldsymbol{E}$ :

$$
0 \longrightarrow i_{2 *} \mathcal{O}_{\mathbb{P}^{1}} \longrightarrow \mathcal{E} \longrightarrow i_{1 *} \mathcal{O}_{\mathbb{P}^{1}}(-2) \longrightarrow 0 .
$$

Because the torsion free sheaves $i_{2 *} \mathcal{O}_{\mathbb{P}^{1}}$ and $i_{1 *} \mathcal{O}_{\mathbb{P}^{1}}(-2)$ have rank one and $\boldsymbol{E}$ is irreducible, they are stable. Because $\varphi\left(i_{2 *} \mathcal{O}_{\mathbb{P}^{1}}\right)=3 / 4$ and $\varphi\left(i_{1 *} \mathcal{O}_{\mathbb{P}^{1}}(-2)\right)=1 / 4$, Theorem 3.10 implies that the HNF 


\section{Burban AND B. Kreußler}

of $\mathcal{E}$ is given by the exact sequence (4). The HN-factors are the two torsion free sheaves of rank one, $i_{2 *} \mathcal{O}_{\mathbb{P}^{1}}$ and $i_{1 *} \mathcal{O}_{\mathbb{P}^{1}}(-2)$, which are not locally free. These are the extreme stable elements with phases $3 / 4$ and $1 / 4$ respectively. Therefore, the indecomposable vector bundle $\mathcal{E}$ is a perfect object of type (iv) which satisfies $\varphi_{-}(\mathcal{E})=1 / 4$ and $\varphi_{+}(\mathcal{E})=3 / 4$.

Remark 4.10. This example shows that the full sub-category of perfect complexes in the category $\mathrm{D}_{\text {coh }}^{\mathrm{b}}(\boldsymbol{E})$ is not closed under taking Harder-Narasimhan factors. We interpret this to be an indication that the derived category of perfect complexes is not an appropriate object for homological mirror symmetry on singular Calabi-Yau varieties.

Remark 4.11. It seems plausible that methods similar to those of this section could be applied to study the derived category of representations of certain derived tame associative algebras. Such may include gentle algebras, skew-gentle algebras and degenerated tubular algebras. The study of Harder-Narasimhan filtrations in conjunction with the action of the group of exact auto-equivalences of the derived category may provide new insight into the combinatorics of indecomposable objects in these derived categories.

Proposition 4.12. Suppose $X, Y \in \mathrm{D}_{\text {coh }}^{\mathrm{b}}(\boldsymbol{E})$ are non-zero.

(i) If $\varphi_{-}(X)<\varphi_{+}(Y)<\varphi_{-}(X)+1$, then $\operatorname{Hom}(X, Y) \neq 0$.

(ii) If $X$ and $Y$ are indecomposable objects which are not of type (i) in Corollary 4.7 and which satisfy $\varphi_{-}(X)=\varphi_{+}(Y)$, then $\operatorname{Hom}(X, Y) \neq 0$.

Proof. If $X$ and $Y$ are semi-stable objects, the claim (i) was proved in Corollary 4.4(iii). Similarly, claim (ii) for two semi-stable objects follows from Corollary 4.4(vi), because there is only one nonperfect object in $\mathrm{P}(\varphi)^{s}$.

For the rest of the proof we treat both cases, (i) and (ii), simultaneously. For the proof of (ii) we keep in mind that Proposition 4.6 implies that no $\mathrm{HN}$-factor has a perfect summand if the object is indecomposable but not semi-stable. If $X \in \mathrm{P}(\varphi)$ is semi-stable but $Y \in \mathrm{D}_{\text {coh }}^{\mathrm{b}}(\boldsymbol{E})$ is arbitrary, we let

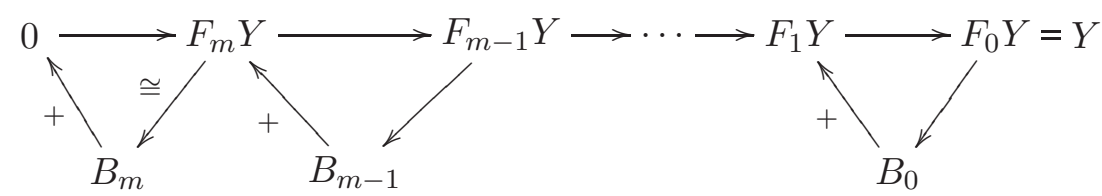

be an HNF of $Y$. As $\varphi\left(B_{m}\right)=\varphi_{+}(Y)$ we know already that $\operatorname{Hom}\left(X, B_{m}\right) \neq 0$. By assumption, we have $\varphi\left(B_{i}[-1]\right)=\varphi\left(B_{i}\right)-1 \leqslant \varphi_{+}(Y)-1<\varphi(X)$. Hence, by Lemma 3.9, $\operatorname{Hom}\left(X, B_{i}[-1]\right)=0$ and the cohomology sequence of the distinguished triangle $F_{i+1} Y \rightarrow F_{i} Y \rightarrow B_{i} \stackrel{+}{\rightarrow}$ provides an inclusion $\operatorname{Hom}\left(X, F_{i+1} Y\right) \subset \operatorname{Hom}\left(X, F_{i} Y\right)$. This implies $0 \neq \operatorname{Hom}\left(X, B_{m}\right) \subset \operatorname{Hom}(X, Y)$.

Finally, in the general case, we let

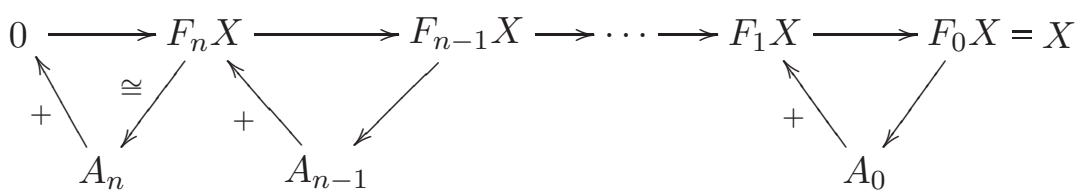

be an HNF of $X$. As $\varphi\left(A_{0}\right)=\varphi_{-}(X)$ we have $\operatorname{Hom}\left(A_{0}, Y\right) \neq 0$. Because

$$
\varphi_{-}\left(F_{1} X[1]\right)=\varphi_{-}\left(F_{1} X\right)+1=\varphi\left(A_{1}\right)+1>\varphi_{-}(X)+1>\varphi_{+}(Y),
$$

Lemma 3.9 implies that $\operatorname{Hom}\left(F_{1} X[1], Y\right)=0$. The distinguished triangle $F_{1} X \rightarrow X \rightarrow A_{0} \stackrel{\leftrightarrow}{\rightarrow}$ gives us now an inclusion $0 \neq \operatorname{Hom}\left(A_{0}, Y\right) \subset \operatorname{Hom}(X, Y)$ and so the claim. 


\section{DERIVED CATEGORIES OF IRREDUCIBLE PROJECTIVE CURVES}

In [Pol02], Polishchuk asked for the classification of all spherical objects in the bounded derived category of a singular projective curve of arithmetic genus one. Below, we shall solve this problem for irreducible curves.

Let $\boldsymbol{E}$ be an irreducible projective curve of arithmetic genus one over our base field $\boldsymbol{k}$. Recall that in this case an object $X \in \mathrm{D}_{\text {coh }}^{\mathrm{b}}(\boldsymbol{E})$ is spherical if

$$
X \text { is perfect and } \operatorname{Hom}(X, X[i]) \cong \begin{cases}k & \text { if } i \in\{0,1\}, \\ 0 & \text { if } i \notin\{0,1\} .\end{cases}
$$

Proposition 4.13. Let $\boldsymbol{E}$ be an irreducible projective curve of arithmetic genus one and $X \in$ $\mathrm{D}_{\text {coh }}^{\mathrm{b}}(\boldsymbol{E})$. Then the following are equivalent:

(i) $X$ is spherical;

(ii) $\operatorname{Hom}(X, X[i]) \cong \begin{cases}\boldsymbol{k} & \text { if } i=0, \\ 0 & \text { if } i=2 \text { or } i<0 ;\end{cases}$

(iii) $X$ is perfect and stable;

(iv) there exists $n \in \mathbb{Z}$ such that $X[n]$ is isomorphic to a simple vector bundle or to a torsion sheaf of length one which is supported at a smooth point of $\boldsymbol{E}$.

In particular, the group of exact auto-equivalences of $\mathrm{D}_{\mathrm{coh}}^{\mathrm{b}}(\boldsymbol{E})$ acts transitively on the set of all spherical objects.

Proof. The implication (i) $\Rightarrow$ (ii) is obvious.

Let us prove (ii) $\Rightarrow$ (iii). First, we observe that $\operatorname{Hom}(X, X) \cong \boldsymbol{k}$ implies that $X$ is indecomposable. Suppose, $X$ is not semi-stable. This is equivalent to $\varphi_{+}(X)>\varphi_{-}(X)$. By Proposition 4.6 we know that all $\mathrm{HN}$-factors of $X$ are extreme. Let $M \geqslant 0$ be the unique integer with $M \leqslant \varphi_{+}(X)-\varphi_{-}(X)<$ $M+1$.

If $M<\varphi_{+}(X)-\varphi_{-}(X)<M+1$, Proposition 4.12(i) implies that $\operatorname{Hom}(X, X[-M]) \neq 0$. Under the assumption (ii), this is possible only if $M=0$. On the other hand, if $M=\varphi_{+}(X)-\varphi_{-}(X)$, we obtain from Proposition 4.12(ii) that $\operatorname{Hom}(X, X[-M]) \neq 0$. Again, this implies $M=0$. So, we have $0<\varphi_{+}(X)-\varphi_{-}(X)<1$.

If we apply the functor $\operatorname{Hom}(\cdot, X)$ to $F_{1} X \stackrel{u}{\rightarrow} X \rightarrow A_{0} \stackrel{+}{\rightarrow}$, the rightmost distinguished triangle of the HNF of $X$, we obtain the exact sequence

$$
\operatorname{Hom}\left(F_{1} X[1], X\right) \rightarrow \operatorname{Hom}\left(A_{0}, X\right) \rightarrow \operatorname{Hom}(X, X) \rightarrow \operatorname{Hom}\left(F_{1} X, X\right),
$$

in which the leftmost term $\operatorname{Hom}\left(F_{1} X[1], X\right)=0$ by Lemma 3.9 , because $\varphi_{-}\left(F_{1} X[1]\right)>\varphi_{-}(X)+1>$ $\varphi_{+}(X)$. The third morphism in this sequence is not the zero map, as it sends $\operatorname{ld}_{X}$ to $u \neq 0$. Because $\operatorname{Hom}(X, X)$ is one-dimensional, this is only possible if $\operatorname{Hom}\left(A_{0}, X\right)=0$. But Proposition 4.12(i) and $\varphi\left(A_{0}\right)<\varphi_{+}(X)<\varphi\left(A_{0}\right)+1$ imply that $\operatorname{Hom}\left(A_{0}, X\right) \neq 0$. This contradiction shows that $X$ must be semi-stable.

We observed earlier that all the JH-factors of an indecomposable semi-stable object are isomorphic to each other. Therefore, any indecomposable semi-stable object which is not stable has a space of endomorphisms of dimension at least two. So, we conclude that $X \in \mathrm{P}(\varphi)^{s}$ for some $\varphi \in \mathbb{R}$.

Because $\operatorname{Hom}(\boldsymbol{k}(s), \boldsymbol{k}(s)[2]) \cong \operatorname{Ext}^{2}(\boldsymbol{k}(s), \boldsymbol{k}(s)) \neq 0$, the transitivity of the action of $\widetilde{\mathrm{SL}}(2, \mathbb{Z})$ on the set $\mathrm{Q}$ implies that none of the extreme stable objects satisfies the condition (ii). Hence, $X$ is perfect and stable.

To prove (iii) $\Rightarrow$ (i), we observe that the group of automorphisms of the curve $\boldsymbol{E}$ acts transitively on the regular locus $\boldsymbol{E} \backslash\{s\}$. Hence, by Proposition 4.2, the group of auto-equivalences of $\mathrm{D}_{\text {coh }}^{\mathrm{b}}(\boldsymbol{E})$ acts transitively on the set of all perfect stable objects. Because, for example, the structure sheaf $\mathcal{O}_{\boldsymbol{E}}$ 


\section{Burban And B. Kreußler}

is spherical, it is now clear that all perfect stable objects are indeed spherical and that the group of exact auto-equivalences of $\mathrm{D}_{\mathrm{coh}}^{\mathrm{b}}(\boldsymbol{E})$ acts transitively on the set of all spherical objects.

To show the equivalence with (iv), it remains to recall that any perfect coherent torsion free sheaf on $\boldsymbol{E}$ is locally free. This follows easily from the Auslander-Buchsbaum formula because we are working in dimension one.

\section{Description of $t$-structures in the case of a singular Weierstraß curve}

The main result of this section is a description of all $t$-structures on the derived category of a singular Weierstraß curve $\boldsymbol{E}$. This generalises results of [GKR04] and [Pol04], where the smooth case was studied. As an application, we obtain a description of the group Aut $\left(\mathrm{D}_{\text {coh }}^{\mathrm{b}}(\boldsymbol{E})\right)$ of all exact auto-equivalences of $\mathrm{D}_{\text {coh }}^{\mathrm{b}}(\boldsymbol{E})$. A second application is a description of Bridgeland's space of stability conditions on $\boldsymbol{E}$.

Recall that a $t$-structure on a triangulated category $D$ is a pair of full subcategories $(D \leqslant 0, D \geqslant 0)$ such that, with the notation $\mathrm{D}^{\geqslant n}:=\mathrm{D}^{\geqslant 0}[-n]$ and $\mathrm{D}^{\leqslant n}:=\mathrm{D}^{\leqslant 0}[-n]$ for any $n \in \mathbb{Z}$, the following hold:

(i) $\mathrm{D} \leqslant 0 \subset \mathrm{D} \leqslant 1$ and $\mathrm{D} \geqslant 1 \subset \mathrm{D} \geqslant 0$;

(ii) $\operatorname{Hom}\left(D^{\leqslant 0}, D \geqslant 1\right)=0$;

(iii) for any object $X \in \mathrm{D}$ there exists a distinguished triangle

$$
A \longrightarrow X \longrightarrow B \stackrel{+}{\longrightarrow}
$$

with $A \in \mathrm{D} \leqslant 0$ and $B \in \mathrm{D} \geqslant 1$.

If $\left(D^{\leqslant 0}, D^{\geqslant 0}\right)$ is a $t$-structure then $A=D \leqslant 0 \cap D \geqslant 0$ has a structure of an Abelian category. It is called the heart of the $t$-structure. In this way, $t$-structures on the derived category $\mathrm{D}_{\text {coh }}^{\mathrm{b}}(\boldsymbol{E})$ lead to interesting Abelian categories embedded into it. The natural $t$-structure on $\mathrm{D}_{\text {coh }}^{\mathrm{b}}(\boldsymbol{E})$ has $\mathrm{D}^{\leqslant n}$ equal to the full subcategory formed by all complexes with non-zero cohomology in degree less than or equal to $n$ only. Similarly, the full subcategory $\mathrm{D} \geqslant n$ consists of all complexes $X$ with $H^{i}(X)=0$ for all $i<n$. The heart of the natural $t$-structure is the Abelian category Coh $_{\boldsymbol{E}}$.

In addition to the natural $t$-structure we also have many interesting $t$-structures on $D_{\text {coh }}^{b}(\boldsymbol{E})$. In order to describe them, we introduce the following notation. We continue to work with the notion of stability and the notation introduced in the previous section. If $\mathrm{P} \subset \mathrm{P}(\theta)^{s}$ is a subset, we denote by $\mathrm{D}[\mathrm{P}, \infty)$ the full subcategory of $\mathrm{D}_{\text {coh }}^{\mathrm{b}}(\boldsymbol{E})$ which is defined as follows: $X \in \mathrm{D}_{\text {coh }}^{\mathrm{b}}(\boldsymbol{E})$ is in $\mathrm{D}[\mathrm{P}, \infty)$ if and only if $X=0$ or all its HN-factors, which have at least one JH-factor which is not in $\mathrm{P}$, have phase $\varphi>\theta$. Similarly, $\mathrm{D}(-\infty, \mathrm{P}]$ denotes the category which is generated by $\mathrm{P}$ and all $\mathrm{P}(\varphi)$ with $\varphi<\theta$. If $\mathrm{P}=\mathrm{P}(\theta)^{s}$ we may abbreviate $\mathrm{D}[\theta, \infty)=\mathrm{D}[\mathrm{P}, \infty)$ and $\mathrm{D}(-\infty, \theta]=\mathrm{D}(-\infty, \mathrm{P}]$. Similarly, if $\mathrm{P}=\emptyset$ we use the abbreviations $\mathrm{D}(\theta, \infty)$ and $\mathrm{D}(-\infty, \theta)$. For any open, closed or half-closed interval $I \subset \mathbb{R}$ we define the full subcategories $\mathrm{D} I$ precisely in the same way. Thus, an object $0 \neq X \in \mathrm{D}_{\text {coh }}^{\mathrm{b}}(\boldsymbol{E})$ is in $\mathrm{D} I$ if and only if $\varphi_{-}(X) \in I$ and $\varphi_{+}(X) \in I$.

Proposition 5.1. Let $\theta \in \mathbb{R}$ and $\mathrm{P}(\theta)^{-} \subset \mathrm{P}(\theta)^{s}$ be arbitrary. Denote by $\mathrm{P}(\theta)^{+}=\mathrm{P}(\theta)^{s} \backslash \mathrm{P}(\theta)^{-}$the complement of $\mathrm{P}(\theta)^{-}$. Then,

$$
\mathrm{D}^{\leqslant 0}:=\mathrm{D}\left[\mathrm{P}(\theta)^{-}, \infty\right)
$$

defines a t-structure on $\mathrm{D}_{\mathrm{coh}}^{\mathrm{b}}(\boldsymbol{E})$ with

$$
\mathrm{D}^{\geqslant 1}:=\mathrm{D}\left(-\infty, \mathrm{P}(\theta)^{+}\right]
$$

The heart $\mathrm{A}\left(\theta, \mathrm{P}(\theta)^{-}\right)$of it is the category $\mathrm{D}\left[\mathrm{P}(\theta)^{-}, \mathrm{P}(\theta)^{+}[1]\right]$, which consists of those objects $X \in$ $\mathrm{D}_{\text {coh }}^{\mathrm{b}}(\boldsymbol{E})$ whose HN-factors either have phase $\varphi \in(\theta, \theta+1)$ or have all its JH-factors in $\mathrm{P}(\theta)^{-}$or $\mathrm{P}(\theta)^{+}[1]$. 


\section{DERIVED CATEGORIES OF IRREDUCIBLE PROJECTIVE CURVES}

Proof. The only non-trivial property which deserves a proof is (iii) in the definition of $t$-structure. Given $X \in \mathrm{D}_{\text {coh }}^{\mathrm{b}}(\boldsymbol{E})$, we have to show that there exists a distinguished triangle $A \rightarrow X \rightarrow B \stackrel{+}{\rightarrow}$ with $A \in \mathrm{D} \leqslant 0$ and $B \in \mathrm{D} \geqslant 1$. In order to construct it, let

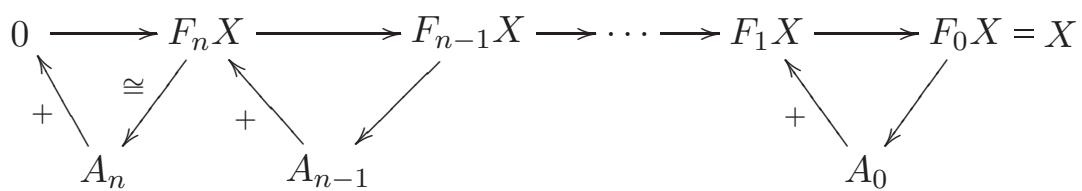

be the HNF of $X$. Because $\varphi\left(A_{i+1}\right)>\varphi\left(A_{i}\right)$ for all $i$, there exists an integer $k, 0 \leqslant k \leqslant n+1$, such that $\varphi\left(A_{k}\right) \geqslant \theta>\varphi\left(A_{k-1}\right)$. If $\varphi\left(A_{k}\right)>\theta$, this implies that $A_{i} \in \mathrm{D} \leqslant 0$ if $i \geqslant k$ and $A_{i} \in \mathrm{D} \geqslant 1$ if $i<k$. In particular, $F_{k} X \in \mathrm{D}^{\leqslant 0}$. In this case, we define $A:=F_{k} X$ and let $A=F_{k} X \rightarrow X$ be the composition of the morphisms in the HNF. If, however, $\varphi\left(A_{k}\right)=\theta$, there is a splitting $A_{k} \cong A_{k}^{-} \oplus A_{k}^{+}$ such that all JH-factors of $A_{k}^{-}$(respectively $A_{k}^{+}$) are in $\mathrm{P}(\theta)^{-}$(respectively $\mathrm{P}(\theta)^{+}$). Now, we apply Lemma 3.4 to the distinguished triangles $F_{k+1} X \stackrel{f}{\longrightarrow} F_{k} X \longrightarrow A_{k} \stackrel{+}{\longrightarrow}$ and $A_{k}^{-} \longrightarrow A_{k} \longrightarrow A_{k}^{+} \stackrel{+}{\longrightarrow}$, given by the splitting of $A_{k}$, to obtain a factorisation $F_{k+1} X \rightarrow A \rightarrow F_{k} X$ of $f$ and the following two distinguished triangles:

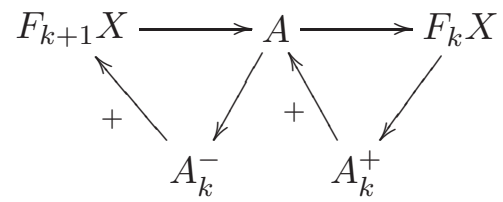

Part of the given HNF of $X$ together with the left one of these two triangles form an HNF of $A$, whence $A \in \mathrm{D} \leqslant 0$. Again, we let $A \rightarrow X$ be obtained by composition with the morphisms in the HNF of $X$. In any case, we choose a distinguished triangle $A \rightarrow X \rightarrow B \stackrel{+}{\rightarrow}$, where $A \rightarrow X$ is the morphism chosen before. From Lemma 3.5 or Remark 3.6 we obtain $B \in \mathrm{D} \geqslant 1$. This proves the proposition.

We shall also need the following standard result.

LemmA 5.2. Let $\left(\mathrm{D}^{\leqslant 0}, \mathrm{D} \geqslant 0\right)$ be a t-structure on a triangulated category. If $X \oplus Y \in \mathrm{D} \leqslant 0$ then $X \in \mathrm{D}^{\leqslant 0}$ and $Y \in \mathrm{D} \leqslant 0$. The corresponding statement holds for $\mathrm{D} \geqslant 0$.

Proof. Let $A \stackrel{f}{\longrightarrow} X \stackrel{g}{\longrightarrow} B \stackrel{+}{\longrightarrow}$ be a distinguished triangle with $A \in \mathrm{D}^{\leqslant 0}$ and $B \in \mathrm{D} \geqslant 1$, which exists due to the definition of a $t$-structure. If $X \notin \mathrm{D} \leqslant 0$, we necessarily have $g \neq 0$ and $B \neq 0$. Because $\operatorname{Hom}\left(\mathrm{D}^{\leqslant 0}, \mathrm{D}^{\geqslant 1}\right)=0$, the composition $X \oplus Y \stackrel{p}{\longrightarrow} X \stackrel{g}{\longrightarrow} B$, in which $p$ denotes the natural projection, must be zero. If $i: X \rightarrow X \oplus Y$ denotes the canonical morphism, we obtain $g=g \circ p \circ i=0$, a contradiction. In the same way it follows that $Y \in \mathrm{D}^{\leqslant 0}$.

Recall that an Abelian category is called Noetherian if any sequence of epimorphisms stabilises. This means that for any sequence of epimorphisms $f_{k}: A_{k} \rightarrow A_{k+1}$ there exists an integer $k_{0}$ such that $f_{k}$ is an isomorphism for all $k \geqslant k_{0}$.

Lemma 5.3. The heart $\mathrm{A}\left(\theta, \mathrm{P}(\theta)^{-}\right)$of the $t$-structure, which was described in Proposition 5.1, is Noetherian if and only if $\mathrm{P}(\theta) \neq\{0\}$ and $\mathrm{P}(\theta)^{-}=\emptyset$. In this case, $\mathrm{A}(\theta, \emptyset)=\mathrm{D}(\theta, \theta+1]$.

Proof. If $\mathrm{P}(\theta)=\{0\}$ then $\mathrm{A}\left(\theta, \mathrm{P}(\theta)^{-}\right)=\mathrm{D}(\theta, \theta+1)$. This category is not Noetherian. To prove this, we follow the proof of Polishchuk in the smooth case [Pol04, Proposition 3.1]. We are going to show, for any non-zero locally free shifted sheaf $E \in \mathrm{D}(\theta, \theta+1)$, the existence of a locally free shifted sheaf $F$ and an epimorphism $E \rightarrow F$ in $\mathrm{D}(\theta, \theta+1)$, which is not an isomorphism. This will be sufficient to show that $\mathrm{D}(\theta, \theta+1)$ is not Noetherian. 


\section{Burban and B. Kreußler}

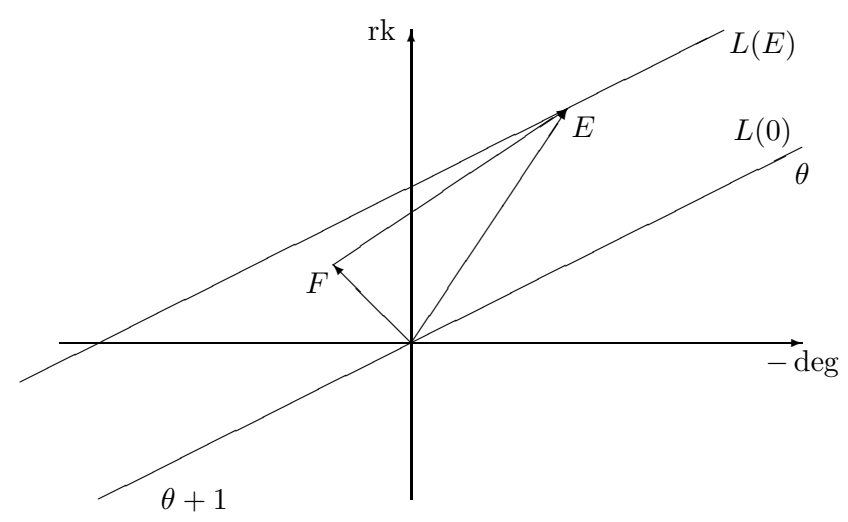

Figure 3. Proof of Lemma 5.3.

By applying an appropriate shift, we may assume $0<\theta<1$. Under this assumption, for every stable coherent sheaf $G$ we have

$$
\begin{aligned}
G \in \mathrm{D}(\theta, \theta+1) & \Longleftrightarrow \theta<\varphi(G) \leqslant 1, \\
G[1] \in \mathrm{D}(\theta, \theta+1) & \Longleftrightarrow 0<\varphi(G)<\theta .
\end{aligned}
$$

For any two objects $X, Y \in \mathrm{D}_{\text {coh }}^{\mathrm{b}}(\boldsymbol{E})$ we define the Euler form to be

$$
\langle X, Y\rangle=\operatorname{rk}(X) \operatorname{deg}(Y)-\operatorname{deg}(X) \operatorname{rk}(Y),
$$

which is the imaginary part of $\overline{Z(X)} Z(Y)$. If $X$ and $Y$ are coherent sheaves and one of them is perfect, we have

$$
\langle X, Y\rangle=\chi(X, Y):=\operatorname{dim} \operatorname{Hom}(X, Y)-\operatorname{dim} \operatorname{Ext}^{1}(X, Y) .
$$

This remains true if we apply arbitrary shifts to the sheaves $X, Y$, where we understand $\chi(X, Y)=$ $\sum_{\nu}(-1)^{\nu} \operatorname{dim} \operatorname{Hom}(X, Y[\nu])$.

Let $E \in \mathrm{D}(\theta, \theta+1)$ be an arbitrary non-zero locally free shifted sheaf. We look at the strip in the plane between the lines $L(0):=\mathbb{R} \exp (i \pi \theta)$ and $L(E):=L(0)+Z(E)$; see Figure 3. This strip must contain lattice points in its interior. Therefore, there exists a lattice point $Z_{F}$ in this strip which enjoys the following properties:

(i) the only lattice points on the closed triangle whose vertices are $0, Z(E), Z_{F}$, are its vertices;

(ii) $\varphi_{F}>\varphi(E)$.

By $\varphi_{F}$ we denote here the unique number which satisfies $\theta<\varphi_{F}<\theta+1$ and $Z_{F} \in \mathbb{R} \exp \left(i \pi \varphi_{F}\right)$. Because $\operatorname{SL}(2, \mathbb{Z})$ acts transitively on $Q$, there exists a stable non-zero locally free shifted sheaf $F \in \mathrm{D}(\theta, \theta+1)$ with $Z(F)=Z_{F}$ and $\varphi(F)=\varphi_{F}$. The assumption $\mathrm{P}(\theta)=\{0\}$ implies $\mathbb{R} \exp (i \pi \theta) \cap$ $\mathbb{Z}^{2}=\{0\}$; hence $Z(E)$ is the only lattice point on the line $L(E)$. This implies that $Z(F)$ is not on the boundary of the strip between $L(0)$ and $L(E)$. In particular, $Z(E)-Z(F)$ is contained in the same half-plane of $L(0)$ as $Z(E)$ and $Z(F)$; see Figure 3. Condition (i) implies $\langle E, F\rangle=1$. Because $E$ is locally free, condition (ii) implies

$$
\operatorname{Ext}^{1}(E, F)=\operatorname{Hom}(F, E)=0 .
$$

Hence, $\operatorname{Hom}(E, F) \cong \boldsymbol{k}$. The evaluation map gives, therefore, a distinguished triangle

$$
\operatorname{Hom}(E, F) \otimes E \rightarrow F \rightarrow T_{E}(F) \stackrel{+}{\longrightarrow}
$$

with $T_{E}(F) \in \mathrm{D}_{\text {coh }}^{\mathrm{b}}(\boldsymbol{E})$. If $C:=T_{E}(F)[-1]$ we obtain a distinguished triangle

$$
C \rightarrow E \rightarrow F \stackrel{+}{\longrightarrow}
$$




\section{DERIVED CATEGORIES OF IRREDUCIBLE PROJECTIVE CURVES}

with $Z(C)=Z(E)-Z(F)$. Because $E$ is a stable non-zero shifted locally free sheaf, it is spherical by Proposition 4.13 and so $T_{E}$ is an equivalence. This implies that $T_{E}(F)$ is spherical and, by Proposition 4.13 again, $C$ is a stable non-zero shifted locally free sheaf. All morphisms in the distinguished triangle (5) are non-zero because $C, E, F$ are indecomposable; see Lemma 3.11. Using Lemma 3.9, this implies that $\theta-1<\varphi(C)<\theta+1$. However, we have seen in which half-plane $Z(C)$ is contained, so that we must have $\theta<\varphi(C)<\theta+1$, which implies that $C \in \mathrm{D}(\theta, \theta+1)$. The distinguished triangle (5) and the definition of the structure of Abelian category on the heart $\mathrm{D}(\theta, \theta+1)$ imply now that the morphism $E \rightarrow F$ in (5) is an epimorphism in $\mathrm{D}(\theta, \theta+1)$. This gives an infinite chain of epimorphisms which are not isomorphisms, so that the category $\mathrm{D}(\theta, \theta+1)$ is indeed not Noetherian.

In order to show that $\mathrm{A}\left(\theta, \mathrm{P}(\theta)^{-}\right)$is not Noetherian for $\mathrm{P}(\theta)^{-} \neq \emptyset$ we may assume $\theta=0$. If there exists a stable element $\boldsymbol{k}(x) \in \mathrm{P}(0)^{-}[1] \subset \mathrm{P}(1)$, where $x \in \boldsymbol{E}$ is a smooth point, we have exact sequences

$$
0 \longrightarrow \mathcal{O}(m x) \longrightarrow \mathcal{O}((m+1) x) \longrightarrow \boldsymbol{k}(x) \longrightarrow 0
$$

in $\operatorname{Coh}_{\boldsymbol{E}}$ with arbitrary $m \in \mathbb{Z}$. Hence the cone of the morphism $\mathcal{O}(m x) \rightarrow \mathcal{O}((m+1) x)$ is isomorphic to $\boldsymbol{k}(x)[0]$. Because $\boldsymbol{k}(x)[0]$ is an object of $\mathrm{D}^{\leqslant-1}$, with regard to the $t$-structure which is defined by $\mathrm{P}(0)^{-}$, we obtain $\tau_{\geqslant 0}(\boldsymbol{k}(x)[0])=0$, which is the cokernel of $\mathcal{O}(m x) \rightarrow \mathcal{O}((m+1) x)$ in the Abelian category $\mathrm{A}\left(0, \mathrm{P}(0)^{-}\right)$; see $[\mathrm{BBD} 82, \S 1.3]$. Hence, there is an exact sequence

$$
0 \longrightarrow \boldsymbol{k}(x)[-1] \longrightarrow \mathcal{O}(m x) \longrightarrow \mathcal{O}((m+1) x) \longrightarrow 0
$$

in $\mathrm{A}\left(0, \mathrm{P}(0)^{-}\right)$and we obtain an infinite chain of epimorphisms

$$
\mathcal{O}(x) \longrightarrow \mathcal{O}(2 x) \longrightarrow \mathcal{O}(3 x) \longrightarrow \cdots
$$

in the category $\mathrm{A}\left(0, \mathrm{P}(0)^{-}\right)$, which, therefore, is not Noetherian. If $\mathrm{P}(0)^{-}[1]$ contains $\boldsymbol{k}(s)$ only, where $s \in \boldsymbol{E}$ is the singular point, we proceed as follows. First, recall that there exist coherent torsion modules with support at $s$ which have finite injective dimension; see for example [BK05, $\S 4]$. To describe examples of them, we can choose a line bundle $\mathcal{L}$ on $\boldsymbol{E}$ and a section $\sigma \in H^{0}(\mathcal{L})$, such that the cokernel of $\sigma: \mathcal{O} \rightarrow \mathcal{L}$ is a coherent torsion module $\mathcal{B}$ of length two with support at $s$. If we embed $\boldsymbol{E}$ into $\mathbb{P}^{2}$, such a line bundle $\mathcal{L}$ is obtained as the tensor product of the restriction of $\mathcal{O}_{\mathbb{P}^{2}}(1)$ with $\mathcal{O}_{\boldsymbol{E}}(-x)$, where $x \in \boldsymbol{E}$ is a smooth point. The section $\sigma$ corresponds to the line in the plane through $x$ and $s$. By twisting with $\mathcal{L}^{\otimes m}$ we obtain exact sequences

$$
0 \longrightarrow \mathcal{L}^{\otimes m} \longrightarrow \mathcal{L}^{\otimes(m+1)} \longrightarrow \mathcal{B} \longrightarrow 0
$$

in $\mathrm{Coh}_{\boldsymbol{E}}$. Because $\mathcal{B}$ is a semi-stable torsion sheaf with support at $s$, all its JH-factors are isomorphic to $\boldsymbol{k}(s)$ and we conclude as above. This completes the proof of Lemma 5.3.

Proposition 5.4. Let $\left(\mathrm{D}^{\leqslant 0}, \mathrm{D} \geqslant 0\right)$ be a $t$-structure on $\mathrm{D}_{\text {coh }}^{\mathrm{b}}(\boldsymbol{E})$ and $B$ a semi-stable indecomposable object in $\mathrm{D}_{\text {coh }}^{\mathrm{b}}(\boldsymbol{E})$. Then either $B \in \mathrm{D}^{\leqslant 0}$ or $B \in \mathrm{D}^{\geqslant 1}$.

Proof. Let $X \stackrel{f}{\rightarrow} B \stackrel{g}{\rightarrow} Y \stackrel{+}{\rightarrow}$ be a distinguished triangle with $X \in \mathrm{D} \leqslant 0$ and $Y \in \mathrm{D} \geqslant 1$. Suppose $X \neq 0$ and $Y \neq 0$ in $\mathrm{D}_{\text {coh }}^{\mathrm{b}}(\boldsymbol{E})$. We decompose both objects into indecomposables $X=\bigoplus X_{i}$ and $Y=\bigoplus Y_{j}$. By Lemma 5.2 we have $X_{i} \in \mathrm{D}^{\leqslant 0}$ and $Y_{j} \in \mathrm{D} \geqslant 1$. If one of the components of the morphisms $Y[-1] \rightarrow X=\bigoplus X_{i}$ or $\bigoplus Y_{j}=Y \rightarrow X$ [1] were zero, by Lemma 3.11 we would obtain a direct summand $X_{i}$ or $Y_{j}$ in $B$. Because $B$ was assumed to be indecomposable, this implies the claim of the proposition.

For the rest of the proof we suppose that all components of these two morphisms are nonzero. This implies that $X_{i}$ and $Y_{j}$ are non-perfect for all $i, j$. Indeed, if $X_{i}$ were perfect, we could apply Serre duality (3) to obtain $\operatorname{Hom}\left(Y, X_{i}[1]\right)=\operatorname{Hom}\left(X_{i}, Y\right)^{*}$, which is zero because $X_{i} \in \mathrm{D} \leqslant 0$ and $Y \in \mathrm{D} \geqslant 1$. The case with perfect $Y_{j}$ can be dealt with similarly. Using Lemma 3.11 again, it 


\section{Burban And B. Kreußler}

follows that none of the components of $f: \bigoplus X_{i} \rightarrow B$ or $g: B \rightarrow \bigoplus Y_{j}$ is zero, because none of the $X_{i}$ could be a direct summand of $Y[-1]$ and none of the $Y_{j}$ could be a summand of $X[1]$. Using Lemma 3.9, this implies $\varphi_{-}\left(X_{i}\right) \leqslant \varphi(B) \leqslant \varphi_{+}\left(Y_{j}\right)$ for all $i, j$. If there exist $i, j$ such that $\varphi_{-}\left(X_{i}\right)-\varphi_{+}\left(Y_{j}\right) \notin \mathbb{Z}$, there exists an integer $k \geqslant 0$ such that $\varphi_{-}\left(X_{i}[k]\right)<\varphi_{+}\left(Y_{j}\right)<\varphi_{-}\left(X_{i}[k]\right)+1$. Using Proposition 4.12(i) this implies that $\operatorname{Hom}\left(X_{i}[k], Y_{j}\right) \neq 0$. But, for any integer $k \geqslant 0$ we have $X_{i}[k] \in \mathrm{D} \leqslant 0$, and because $Y_{j} \in \mathrm{D}^{\geqslant 1}$, we should have $\operatorname{Hom}\left(X_{i}[k], Y_{j}\right)=0$. This contradiction implies that $\varphi_{-}\left(X_{i}\right)-\varphi_{+}\left(Y_{j}\right) \in \mathbb{Z}$ for all $i, j$. But, if $k=\varphi_{+}\left(Y_{j}\right)-\varphi_{-}\left(X_{i}\right)$, we still have $\operatorname{Hom}\left(X_{i}[k], Y_{j}\right) \neq 0$, which follows from Proposition 4.12(ii) because $X_{i}$ and $Y_{j}$ are not perfect. The conclusion is now that we must have $X=0$ or $Y=0$, which implies the claim.

Lemma 5.5. Let $\left(\mathrm{D}^{\leqslant 0}, \mathrm{D}^{\geqslant 0}\right)$ be a $t$-structure on $\mathrm{D}_{\text {coh }}^{\mathrm{b}}(\boldsymbol{E})$. If $F \in \mathrm{D}^{\leqslant 0}$ and $G \in \mathrm{D} \geqslant 1$, then $\varphi_{-}(F) \geqslant$ $\varphi_{+}(G)$.

Proof. Suppose $\varphi_{-}(F)<\varphi_{+}(G)$. It is sufficient to derive a contradiction for indecomposable objects $F$ and $G$. Because, for any $k \geqslant 0, F[k] \in \mathrm{D} \leqslant 0$, we may replace $F$ by $F[k]$ and can assume that $0<\varphi_{+}(G)-\varphi_{-}(F) \leqslant 1$. Now, there exists a stable vector bundle $\mathcal{B}$ on $\boldsymbol{E}$ and an integer $r$ such that

$$
\varphi_{-}(F)<\varphi(\mathcal{B}[r])<\varphi_{+}(G) \leqslant \varphi_{-}(F)+1 .
$$

By Proposition 5.4, $\mathcal{B}[r]$ is in $\mathrm{D}^{\leqslant 0}$ or in $\mathrm{D} \geqslant 1$. But, from Proposition 4.12(i) we deduce that $\operatorname{Hom}(F, \mathcal{B}[r]) \neq 0$ and $\operatorname{Hom}(\mathcal{B}[r], G) \neq 0$. If $\mathcal{B}[r] \in \mathrm{D} \geqslant 1$, the first inequality contradicts $F \in \mathrm{D}^{\leqslant 0}$, and if $\mathcal{B}[r] \in \mathrm{D} \leqslant 0$, the second one contradicts $G \in \mathrm{D} \geqslant 1$.

Theorem 5.6. Let $\left(\mathrm{D}^{\leqslant 0}, \mathrm{D}^{\geqslant 0}\right)$ be a t-structure on $\mathrm{D}_{\text {coh }}^{\mathrm{b}}(\boldsymbol{E})$. Then there exist a number $\theta \in \mathbb{R}$ and a subset $\mathrm{P}(\theta)^{-} \subset \mathrm{P}(\theta)^{s}$, such that

$$
\mathrm{D}^{\leqslant 0}=\mathrm{D}\left[\mathrm{P}(\theta)^{-}, \infty\right) \quad \text { and } \quad \mathrm{D}^{\geqslant 1}=\mathrm{D}\left(-\infty, \mathrm{P}(\theta)^{+}\right] .
$$

Proof. From Lemma 5.5 we deduce the existence of $\theta \in \mathbb{R}$ such that $\mathrm{D}(\theta, \infty) \subset \mathrm{D} \leqslant 0$ and $\mathrm{D}(-\infty, \theta) \subset$ $D^{\geqslant 1}$. If we define $P(\theta)^{-}=P(\theta)^{s} \cap D^{\leqslant 0}$ and $P(\theta)^{+}=P(\theta)^{s} \cap D^{\geqslant 1}$, Proposition 5.4 implies that $P(\theta)^{s}=$ $\mathrm{P}(\theta)^{-} \cup \mathrm{P}(\theta)^{+}$. Hence, $\mathrm{D}\left[\mathrm{P}(\theta)^{-}, \infty\right) \subset \mathrm{D}^{\leqslant 0}$ and $\mathrm{D}\left(-\infty, \mathrm{P}(\theta)^{+}\right] \subset \mathrm{D}^{\geqslant 1}$. From Proposition 5.1 we know that $\left(\mathrm{D}\left[\mathrm{P}(\theta)^{-}, \infty\right), \mathrm{D}\left(-\infty, \mathrm{P}(\theta)^{+}[1]\right]\right)$ defines a $t$-structure. Now, the statement of the theorem follows.

Remark 5.7. In the case of a smooth elliptic curve, Theorem 5.6 was proved in [GKR04]. If $\theta \notin \mathrm{Q}$ the heart $\mathrm{D}(\theta, \theta+1)$ of the corresponding $t$-structure is a finite-dimensional non-Noetherian Abelian category of infinite global dimension. In the smooth case, such a heart corresponds to the category of holomorphic vector bundles on a non-commutative torus in the sense of Polishchuk and Schwarz [PS03]. It is an interesting problem to find a similar interpretation of these Abelian categories in the case of a singular Weierstraß curve $\boldsymbol{E}$.

To complete this section we give two applications of Theorem 5.6. The first is a description of the group of exact auto-equivalences of the triangulated category $\mathrm{D}_{\mathrm{coh}}^{\mathrm{b}}(\boldsymbol{E})$. The second application is a description of Bridgeland's space of all stability structures on $D_{\text {coh }}^{\mathrm{b}}(\boldsymbol{E})$. In both cases, $\boldsymbol{E}$ is an irreducible curve of arithmetic genus one over $\boldsymbol{k}$.

COROLlary 5.8. There exists an exact sequence of groups

$$
\mathbf{1} \longrightarrow \operatorname{Aut}^{0}\left(\mathrm{D}_{\text {coh }}^{\mathrm{b}}(\boldsymbol{E})\right) \longrightarrow \operatorname{Aut}\left(\mathrm{D}_{\text {coh }}^{\mathrm{b}}(\boldsymbol{E})\right) \longrightarrow \mathrm{SL}(2, \mathbb{Z}) \longrightarrow \mathbf{1}
$$

in which $\operatorname{Aut}^{0}\left(\mathrm{D}_{\text {coh }}^{\mathrm{b}}(\boldsymbol{E})\right)$ is generated by tensor products with line bundles of degree zero, automorphisms of the curve and the shift by 2 .

Proof. The homomorphism Aut $\left(\mathrm{D}_{\text {coh }}^{\mathrm{b}}(\boldsymbol{E})\right) \rightarrow \mathrm{SL}(2, \mathbb{Z})$ is defined by describing the action of an autoequivalence on $\mathbf{K}(\boldsymbol{E})$ in terms of the coordinate functions (deg, rk). That this is indeed in $\operatorname{SL}(2, \mathbb{Z})$ 


\section{DERIVED CATEGORIES OF IRREDUCIBLE PROJECTIVE CURVES}

follows, for example, because $\operatorname{Aut}\left(\mathrm{D}_{\mathrm{coh}}^{\mathrm{b}}(\boldsymbol{E})\right)$ preserves stability and the Euler form

$$
\begin{aligned}
\langle\mathcal{F}, \mathcal{G}\rangle & =\operatorname{dim} \operatorname{Hom}(\mathcal{F}, \mathcal{G})-\operatorname{dim} \operatorname{Hom}(\mathcal{G}, \mathcal{F}) \\
& =\operatorname{rk}(\mathcal{F}) \operatorname{deg}(\mathcal{G})-\operatorname{deg}(\mathcal{F}) \operatorname{rk}(\mathcal{G})
\end{aligned}
$$

for stable and perfect sheaves $\mathcal{F}, \mathcal{G}$. Clearly, tensor products with line bundles of degree zero, automorphisms of the curve and the shift by 2 are contained in the kernel of this homomorphism. In order to show that the kernel coincides with $\operatorname{Aut}^{0}\left(D_{\text {coh }}^{\mathrm{b}}(\boldsymbol{E})\right)$, we let $\mathbb{G}$ be an arbitrary exact auto-equivalence of $\mathrm{D}_{\text {coh }}^{\mathrm{b}}(\boldsymbol{E})$. Then, $\mathbb{G}\left(\mathrm{Coh}_{\boldsymbol{E}}\right)$ is still Noetherian and it is the heart of the $t$-structure $\left(\mathbb{G}\left(D^{\leqslant 0}\right), \mathbb{G}\left(D^{\geqslant 0}\right)\right)$. From Theorem 5.6 and Lemma 5.3 we know all Noetherian hearts of $t$-structures. We obtain $\mathbb{G}\left(\operatorname{Coh}_{\boldsymbol{E}}\right)=\mathrm{D}(\theta, \theta+1]$ with $\mathrm{P}(\theta) \neq\{0\}$. Now, by Corollary 4.4 there exists $\Phi \in \widetilde{\mathrm{SL}}(2, \mathbb{Z})$ which maps $\mathrm{D}(\theta, \theta+1]$ to $\mathrm{D}(0,1]=\mathrm{Coh}_{\boldsymbol{E}}$. This implies that the auto-equivalence $\Phi \circ \mathbb{G}$ induces an auto-equivalence of the category $\mathrm{Coh}_{\boldsymbol{E}}$. It is well known that such an auto-equivalence has the form $f^{*}(\mathcal{L} \otimes \cdot)$, where $f: \boldsymbol{E} \rightarrow \boldsymbol{E}$ is an isomorphism and $\mathcal{L}$ is a line bundle. Note that $f^{*}(\mathcal{L} \otimes \cdot)$ is sent to the identity in $\operatorname{SL}(2, \mathbb{Z})$ if and only if $\mathcal{L}$ is of degree zero. The composition of $\Phi \circ \mathbb{G}$ with the inverse of $f^{*}(\mathcal{L} \otimes \cdot)$ satisfies the assumptions of [BO97, Proposition A.3], hence is isomorphic to the identity. Because the kernel of the homomorphism $\widetilde{\mathrm{SL}}(2, \mathbb{Z}) \rightarrow \operatorname{SL}(2, \mathbb{Z})$, which is induced by the action of $\widetilde{\mathrm{SL}}(2, \mathbb{Z})$ on $\mathrm{D}_{\text {coh }}^{\mathrm{b}}(\boldsymbol{E})$ and the above homomorphism $\operatorname{Aut}\left(\mathrm{D}_{\text {coh }}^{\mathrm{b}}(\boldsymbol{E})\right) \longrightarrow \mathrm{SL}(2, \mathbb{Z})$, is generated by the element of $\widetilde{\mathrm{SL}}(2, \mathbb{Z})$ which acts as the shift by 2 , the claim now follows.

For our second application, we recall Bridgeland's definition of stability condition on a triangulated category [Bri02].

Recall that we set $\mathrm{K}(\boldsymbol{E})=\mathrm{K}_{0}(\operatorname{Coh}(\boldsymbol{E})) \cong \mathrm{K}_{0}\left(\mathrm{D}_{\text {coh }}^{\mathrm{b}}(\boldsymbol{E})\right)$. Following Bridgeland [Bri02], we call a pair $(W, \mathrm{R})$ a stability condition on $\mathrm{D}_{\text {coh }}^{\mathrm{b}}(\boldsymbol{E})$ if

$$
W: \mathrm{K}(\boldsymbol{E}) \longrightarrow \mathbb{C}
$$

is a group homomorphism and $\mathrm{R}$ is a compatible slicing of $\mathrm{D}_{\text {coh }}^{\mathrm{b}}(\boldsymbol{E})$. A slicing $\mathrm{R}$ consists of a collection of full additive sub-categories $\mathrm{R}(t) \subset \mathrm{D}_{\text {coh }}^{\mathrm{b}}(\boldsymbol{E}), t \in \mathbb{R}$, such that:

(i) $\forall t \in \mathbb{R}, \quad \mathrm{R}(t+1)=\mathrm{R}(t)[1]$;

(ii) if $t_{1}>t_{2}$ and $A_{\nu} \in \mathrm{R}\left(t_{\nu}\right)$, then $\operatorname{Hom}\left(A_{1}, A_{2}\right)=0$;

(iii) each non-zero object $X \in \mathrm{D}_{\text {coh }}^{\mathrm{b}}(\boldsymbol{E})$ has an $\mathrm{HNF}$

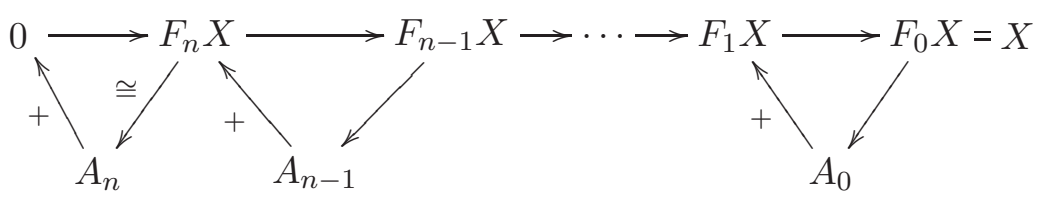

in which $0 \neq A_{\nu} \in \mathrm{R}\left(\varphi_{\nu}\right)$ and $\varphi_{n}>\varphi_{n-1}>\cdots>\varphi_{1}>\varphi_{0}$.

Compatibility means, for all non-zero $A \in \mathrm{R}(t)$,

$$
W(A) \in \mathbb{R}_{>0} \exp (i \pi t)
$$

By $\varphi^{\mathrm{R}}$ we denote the phase function on R-semi-stable objects. Similarly, we denote by $\varphi_{+}^{\mathrm{R}}(X)$ and $\varphi_{-}^{\mathrm{R}}(X)$ the largest, respectively smallest, phase of an R-HN-factor of $X$.

The standard stability condition, which was studied in the previous section, will always be denoted by $(Z, \mathrm{P})$. This stability condition has a slicing which is locally finite; see [Bri02, Definition 5.7]. A slicing $\mathrm{R}$ is called locally finite if and only if there exists $\eta>0$ such that for any $t \in \mathbb{R}$ the quasi-Abelian category $\mathrm{D}^{\mathrm{R}}(t-\eta, t+\eta)$ is of finite length, i.e. Artinian and Noetherian. This category consists of those objects $X \in \mathrm{D}_{\text {coh }}^{\mathrm{b}}(\boldsymbol{E})$ which satisfy $t-\eta<\varphi_{-}^{\mathrm{R}}(X) \leqslant \varphi_{+}^{\mathrm{R}}(X)<t+\eta$.

In order to obtain a good moduli space of stability conditions, Bridgeland [Bri02] requires the stability conditions to be numerical. This means that the central charge $W$ factors through 


\section{Burban and B. Kreußler}

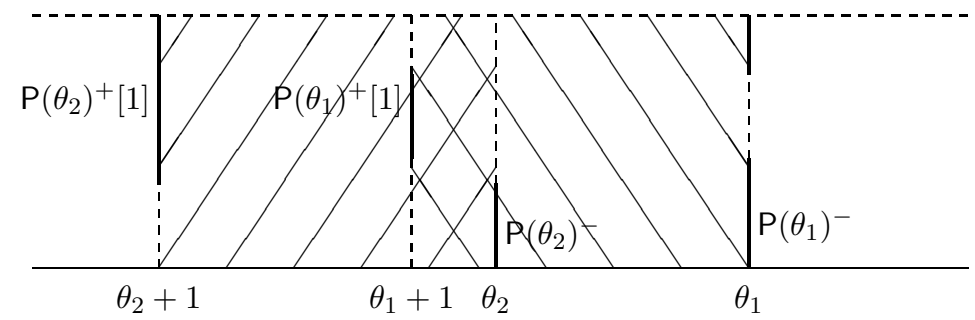

FiguRE 4. Intersection of two hearts.

the numerical Grothendieck group. This makes sense if, for any two objects $E, F$ of the triangulated category in question, the vector spaces $\bigoplus_{i} \operatorname{Hom}(E, F[i])$ are finite-dimensional. This condition is not satisfied for $\mathrm{D}_{\text {coh }}^{\mathrm{b}}(\boldsymbol{E})$ if $\boldsymbol{E}$ is singular. However, in the case of our interest, we do not need such an extra condition, because the Grothendieck group $\mathrm{K}(\boldsymbol{E})$ is sufficiently small. From Lemma 3.1 we know that $\mathrm{K}(\boldsymbol{E}) \cong \mathbb{Z}^{2}$ with generators $\left[\mathcal{O}_{\boldsymbol{E}}\right]$ and $[\boldsymbol{k}(x)], x \in \boldsymbol{E}$ arbitrary. Because $Z(\boldsymbol{k}(x))=-1$ and $Z\left(\mathcal{O}_{\boldsymbol{E}}\right)=i$, it is now clear that any homomorphism $W: \mathbf{K}(\boldsymbol{E}) \rightarrow \mathbb{C}$ can be written as $W(E)=w_{1} \operatorname{deg}(E)+w_{2} \operatorname{rk}(E)$ with $w_{1}, w_{2} \in \mathbb{C}$. Equivalently, if we identify $\mathbb{C}$ with $\mathbb{R}^{2}$, there exists a $2 \times 2$ matrix $A$ such that $W=A \circ Z$.

Definition 5.9. By Stab $\boldsymbol{E}$ we denote the set of all stability conditions $(W, \mathrm{R})$ on $\mathrm{D}_{\text {coh }}^{\mathrm{b}}(\boldsymbol{E})$ for which $\mathrm{R}$ is a locally finite slicing.

Lemma 5.10. For any $(W, \mathrm{R}) \in \operatorname{Stab}(\boldsymbol{E})$ there exists a matrix $A \in \mathrm{GL}(2, \mathbb{R})$, such that $W=A \circ Z$.

Proof. As seen above, there exists a not necessarily invertible matrix $A$ such that $W=A \circ Z$. If $A$ were not invertible, there would exist a number $t_{0} \in \mathbb{R}$ such that $W(\mathrm{~K}(\boldsymbol{E})) \subset \mathbb{R} \exp \left(i \pi t_{0}\right)$. This implies that there may exist a non-zero object in $\mathrm{R}(t)$ only if $t-t_{0} \in \mathbb{Z}$. The assumption that the slicing $\mathrm{R}$ is locally finite implies now that $\mathrm{R}(t)$ is of finite length for any $t \in \mathbb{R}$. On the other hand, the heart of the $t$-structure, which is defined by $(W, \mathrm{R})$, is $\mathrm{R}\left(t_{0}\right)$ up to a shift. However, in Lemma 5.3 we determined all Noetherian hearts of $t$-structures on $D_{\text {coh }}^{\mathrm{b}}(\boldsymbol{E})$ and none of them is Artinian. This contradiction shows that $A$ is invertible.

Lemma 5.11. If $(W, \mathrm{R}) \in \operatorname{Stab}(\boldsymbol{E})$, there exists a unique strictly increasing function $f: \mathbb{R} \rightarrow \mathbb{R}$ with $f(t+1)=f(t)+1$ and $\mathrm{R}(t)=\mathrm{P}(f(t))$.

Proof. By definition, $W(\mathrm{R}(t)) \subset \mathbb{R}_{>0} \exp (i \pi t)$. By Lemma 5.10, there exists a linear isomorphism $A$ such that $W=A^{-1} \circ Z$. This implies that there is a function $f: \mathbb{R} \rightarrow \mathbb{R}$ such that $Z(\mathrm{R}(t)) \subset$ $\mathbb{R}_{>0} \exp (i \pi f(t))$. On the other hand, $\mathrm{R}(t)$ is the intersection of two hearts of $t$-structures. By Proposition 5.1 these hearts are of the form $\mathrm{D}\left[\mathrm{P}\left(\theta_{1}\right)^{-}, \mathrm{P}\left(\theta_{1}\right)^{+}[1]\right]$ and $\mathrm{D}\left[\mathrm{P}\left(\theta_{2}\right)^{-}, \mathrm{P}\left(\theta_{2}\right)^{+}[1]\right]$ with $\theta_{1} \leqslant \theta_{2}$. These have non-empty intersection only if $\theta_{2} \leqslant \theta_{1}+1$. Their intersection is contained in $\mathrm{D}\left[\theta_{2}, \theta_{1}+1\right]$; see Figure 4.

Moreover, if $\theta_{2}<\theta_{1}+1$, there exist $\alpha, \beta \in \mathrm{Q}$ with $\theta_{2}<\alpha<\beta<\theta_{1}+1 \leqslant \theta_{2}+1$. In this case we have two non-trivial sub-categories $\mathrm{P}(\alpha) \subset \mathrm{R}(t)$ and $\mathrm{P}(\beta) \subset \mathrm{R}(t)$. However, because $0<\beta-\alpha<1$ and $Z(\mathrm{R}(t)) \subset \mathbb{R}_{>0} \exp (i \pi f(t))$, we cannot have $Z(\mathrm{P}(\alpha)) \subset \mathbb{R}_{>0} \exp (i \pi \alpha)$ and $Z(\mathrm{P}(\beta)) \subset \mathbb{R}_{>0} \exp (i \pi \beta)$. Hence, $\theta_{2}=\theta_{1}+1=f(t)$ and we obtain $\mathrm{R}(t) \subset \mathrm{P}(f(t))$. From $\mathrm{R}(t+m)=$ $\mathrm{R}(t)[m]$ we easily obtain $f(t+m)=f(t)+m$. Moreover, $f\left(t_{2}\right)=f\left(t_{1}\right)+m$ with $m \in \mathbb{Z}$ implies that $t_{2}-t_{1} \in \mathbb{Z}$, because the image of $W$ is not contained in a line by Lemma 5.10 .

Next, we show that $f$ is strictly increasing. Suppose that $t_{1}<t_{2}, t_{2}-t_{1} \notin \mathbb{Z}$ and both $\mathrm{R}\left(t_{i}\right)$ contain non-zero objects $X_{i}$. For any $m \geqslant 0$ we have $\operatorname{Hom}\left(X_{2}, X_{1}[-m]\right)=0$. If $f\left(t_{2}\right)<f\left(t_{1}\right)$, we choose $m \geqslant 0$ such that $f\left(t_{2}\right)<f\left(t_{1}\right)-m<f\left(t_{2}\right)+1$ and obtain $X_{2} \in \mathrm{P}\left(f\left(t_{2}\right)\right)$ and $X_{1}[-m] \in$ $\mathrm{P}\left(f\left(t_{1}\right)-m\right)$. But this implies, by Corollary 4.4(iii), that $\operatorname{Hom}\left(X_{2}, X_{1}[-m]\right) \neq 0$, a contradiction. 


\section{DERIVED CATEGORIES OF IRREDUCIBLE PROJECTIVE CURVES}

Hence, we have shown that $f$ is strictly increasing with $f(t+1)=f(t)+1$ and $\mathrm{R}(t) \subset \mathrm{P}(f(t))$. In particular, any R-HNF is a P-HNF as well. Therefore, all P-semi-stable objects are R-semi-stable and we obtain $\mathrm{R}(t)=\mathrm{P}(f(t))$.

It was shown in [Bri02] that the group $\widetilde{G L}^{+}(2, \mathbb{R})$ acts naturally on the moduli space of stability conditions $\operatorname{Stab}(\boldsymbol{E})$. This group is the universal cover of $\mathrm{GL}^{+}(2, \mathbb{R})$ and has the following description:

$$
\widetilde{\mathrm{GL}}^{+}(2, \mathbb{R})=\left\{(A, f) \mid A \in \mathrm{GL}^{+}(2, \mathbb{R}), f: \mathbb{R} \rightarrow \mathbb{R} \text { compatible }\right\},
$$

where compatibility means that $f$ is strictly increasing, satisfies $f(t+1)=f(t)+1$ and induces the same map on $S^{1} \cong \mathbb{R} / 2 \mathbb{Z}$ as $A$ does on $S^{1} \cong \mathbb{R}^{2} \backslash\{0\} / \mathbb{R}^{*}$. The action is simply $(A, f) \cdot(W, \mathrm{Q})=$ $\left(A^{-1} \circ W, \mathrm{Q} \circ f\right)$. So, this action basically is a relabelling of the slices. The following result generalises [Bri02, Theorem 9.1], to the singular case.

Proposition 5.12. The action of $\widetilde{\mathrm{GL}}^{+}(2, \mathbb{R})$ on $\operatorname{Stab}(\boldsymbol{E})$ is simply transitive.

Proof. If $(W, \mathrm{R}) \in \operatorname{Stab}(\boldsymbol{E})$, the two values $W\left(\mathcal{O}_{\boldsymbol{E}}\right)$ and $W\left(\boldsymbol{k}\left(p_{0}\right)\right)$ determine a linear transformation $A^{-1} \in \mathrm{GL}(2, \mathbb{R})$ such that $W=A^{-1} \circ Z$ (see Lemma 5.10). By construction, the function $f: \mathbb{R} \rightarrow \mathbb{R}$ of Lemma 5.11 induces the same mapping on $S^{1} \cong \mathbb{R} / 2 \mathbb{Z}$ as $A^{-1}$ does on $S^{1} \cong \mathbb{R}^{2} \backslash\{0\} / \mathbb{R}^{*}$. Therefore, $A \in \mathrm{GL}^{+}(2, \mathbb{R})$ and we obtain $(A, f) \in \widetilde{\mathrm{GL}}^{+}(2, \mathbb{R})$ which satisfies $(W, \mathrm{R})=(A, f) \cdot(Z, \mathrm{P})$. Finally, if $(A, f) \cdot(Z, \mathrm{P})=(Z, \mathrm{P})$ for some $(A, f) \in \widetilde{\mathrm{GL}}^{+}(2, \mathbb{R})$, we obtain $f(t)=t$ for all $t \in \mathbb{R}$. This implies easily that $A=\mathbf{1}$.

The group $\operatorname{Aut}\left(\mathrm{D}_{\text {coh }}^{\mathrm{b}}(\boldsymbol{E})\right)$ acts on $\operatorname{Stab}(\boldsymbol{E})$ by the rule

$$
\mathbb{G} \cdot(W, \mathrm{R}):=(\overline{\mathbb{G}} \circ W, \mathbb{G}(\mathrm{R})) .
$$

Here, $\overline{\mathbb{G}} \in \mathrm{SL}(2, \mathbb{Z})$ is the image of $\mathbb{G} \in \operatorname{Aut}\left(\mathrm{D}_{\text {coh }}^{\mathrm{b}}(\boldsymbol{E})\right)$ under the homomorphism of Corollary 5.8 and $\mathbb{G}(\mathrm{R})(t):=\mathbb{G}(\mathrm{R}(t))$. Because automorphisms of $\boldsymbol{E}$ and twists by line bundles act trivially on $\operatorname{Stab}(\boldsymbol{E})$, we obtain

$$
\operatorname{Stab}(\boldsymbol{E}) / \operatorname{Aut}\left(\mathrm{D}_{\text {coh }}^{\mathrm{b}}(\boldsymbol{E})\right) \cong \mathrm{GL}^{+}(2, \mathbb{R}) / \mathrm{SL}(2, \mathbb{Z})
$$

which is a $\mathbb{C}^{\times}$-bundle over the coarse moduli space of elliptic curves. This result coincides with Bridgeland's result in the smooth case. The main reason for this coincidence seems to be the irreducibility of the curve. Example 5.14 below shows that the situation is significantly more difficult in the case of reducible degenerations of elliptic curves.

Remark 5.13. Our results show that singular and smooth Weierstraß curves $\boldsymbol{E}$ share the following properties.

(i) A coherent sheaf $\mathcal{F}$ is stable if and only if $\operatorname{End}(\mathcal{F}) \cong \boldsymbol{k}$.

(ii) Any spherical object is a shift of a stable vector bundle or of a structure sheaf $\boldsymbol{k}(x)$ of a smooth point $x \in \boldsymbol{E}$.

(iii) The category of semi-stable sheaves of a fixed slope is equivalent to the category of coherent torsion sheaves. Such an equivalence is induced by an auto-equivalence of $D_{\text {coh }}^{\mathrm{b}}(\boldsymbol{E})$.

(iv) There is an exact sequence of groups $\mathbf{1} \rightarrow\left\langle\operatorname{Aut}(\boldsymbol{E}), \operatorname{Pic}^{0}(\boldsymbol{E}),[2]\right\rangle \rightarrow \operatorname{Aut}\left(\mathrm{D}_{\text {coh }}^{\mathrm{b}}(\boldsymbol{E})\right) \rightarrow \mathrm{SL}(2, \mathbb{Z}) \rightarrow$ 1.

(v) $\widetilde{\mathrm{GL}}^{+}(2, \mathbb{R})$ acts transitively on $\operatorname{Stab}(\boldsymbol{E})$.

(vi) $\operatorname{Stab}(\boldsymbol{E}) / \operatorname{Aut}\left(\mathrm{D}_{\text {coh }}^{\mathrm{b}}(\boldsymbol{E})\right) \cong \mathrm{GL}^{+}(2, \mathbb{R}) / \mathrm{SL}(2, \mathbb{Z})$.

Example 5.14. Let $C_{2}$ denote a reducible curve which has two components, both isomorphic to $\mathbb{P}^{1}$ and which intersect transversally at two distinct points. This curve has arithmetic genus one and appears as a degeneration of a smooth elliptic curve. On this curve, there exists a line bundle $\mathcal{L}$ 


\section{Burban And B. Kreußler}

which fails to be stable with respect to some stability conditions. To construct an explicit example, denote by $\pi: \widetilde{C}_{2} \rightarrow C_{2}$ the normalisation, so that $\widetilde{C}_{2}$ is the disjoint union of two copies of $\mathbb{P}^{1}$. There is a $\boldsymbol{k}^{\times}$-family of line bundles whose pull-back to $\widetilde{C}_{2}$ is $\mathcal{O}_{\mathbb{P}^{1}}$ on one component and $\mathcal{O}_{\mathbb{P}^{1}}(2)$ on the other. The element in $\boldsymbol{k}^{\times}$corresponds to a gluing parameter over one of the two singularities. Let $\mathcal{L}$ denote one such line bundle. If $i_{\nu}: \mathbb{P}^{1} \rightarrow C_{2}, \nu=1,2$, denote the embeddings of the two components, we fix notation so that $i_{1}^{*} \mathcal{L} \cong \mathcal{O}_{\mathbb{P}^{1}}$ and $i_{2}^{*} \mathcal{L} \cong \mathcal{O}_{\mathbb{P}^{1}}(2)$. There is an exact sequence of coherent sheaves on $C_{2}$,

$$
0 \longrightarrow i_{2 *} \mathcal{O}_{\mathbb{P} 1} \longrightarrow \mathcal{L} \longrightarrow i_{1 *} \mathcal{O}_{\mathbb{P} 1} \longrightarrow 0 .
$$

Moreover, the only non-trivial quotients of $\mathcal{L}$ are $\mathcal{L} \rightarrow i_{1 *} \mathcal{O}_{\mathbb{P}^{1}}$ and $\mathcal{L} \rightarrow i_{2 *} \mathcal{O}_{\mathbb{P}^{1}}(2)$.

For arbitrary positive real numbers $a, b$ we define a centred slope-function $W_{a, b}$ on the category $\mathrm{Coh}_{C_{2}}$ by

$$
W_{a, b}(F):=-\operatorname{deg}(F)+i\left(a \cdot \operatorname{rk}\left(i_{1}^{*} F\right)+b \cdot \operatorname{rk}\left(i_{2}^{*} F\right)\right),
$$

where $\operatorname{deg}(F)=h^{0}(F)-h^{1}(F)$. For example,

$$
W_{a, b}\left(i_{1 *} \mathcal{O}_{\mathbb{P}^{1}}(d)\right)=-d-1+i a \quad \text { and } \quad W_{a, b}\left(i_{2 *} \mathcal{O}_{\mathbb{P}^{1}}(d)\right)=-d-1+i b .
$$

Using the exact sequence $(7)$, we obtain $W_{a, b}(\mathcal{L})=-2+i(a+b)$. Using results of [Rud97], it is easy to see that $W_{a, b}$ has the Harder-Narasimhan property in the sense of [Bri02]. Hence, by [Bri02, Proposition 5.3], $W_{a, b}$ defines a stability condition on $\mathrm{D}_{\text {coh }}^{\mathrm{b}}\left(C_{2}\right)$. With respect to this stability condition, the line bundle $\mathcal{L}$ is stable precisely when $2 /(a+b)<1 / a$, which is equivalent to $a<b$. It is semi-stable, but not stable, if $b=a$. If $a>b, \mathcal{L}$ is not even semi-stable.

This example illustrates an interesting effect, which was not available on an irreducible curve of arithmetic genus one. It is an interesting problem to describe the subset in $\operatorname{Stab}(\boldsymbol{E})$ for which a given line bundle $\mathcal{L}$ is semi-stable. This is a closed subset; see [Bri02]. Physicists call the boundary of this set the line of marginal stability; see e.g. [AD02]. The example above describes the intersection of this set with a two-parameter family of stability conditions in $\operatorname{Stab}(\boldsymbol{E})$.

Remark 5.15. In the case of an irreducible curve of arithmetic genus one, we have shown in Proposition 4.13 that Aut $\left(\mathrm{D}_{\text {coh }}^{\mathrm{b}}(\boldsymbol{E})\right)$ acts transitively on the set of all spherical objects on $\boldsymbol{E}$. Polishchuk [Pol02] conjectured that this is likewise true in the case of reducible curves with trivial dualising sheaf. However, on the curve $C_{2}$ there exists a spherical complex which has non-zero cohomology in two different degrees; see [BB05]. This indicates that the reducible case is more difficult and involves new features.

\section{ACKNowledgements}

I. B. would like to thank the Max-Planck-Institut für Mathematik in Bonn for financial support. Both I. B. and B. K. would like to thank Yuriy Drozd, Daniel Huybrechts, Bernhard Keller, Raphaël Rouquier and Olivier Schiffmann for helpful discussions, and the referee for his or her constructive comments.

\section{REFERENCES}

AD02 P. Aspinwall and M. Douglas, D-brane stability and monodromy, J. High Energy Phys. 5 (2002), no. 31,35 pp., hep-th/0110071.

Ati57 M. Atiyah, Vector bundles over an elliptic curve, Proc. London Math. Soc. (3) 7 (1957), 414-452.

BBHM02 C. Bartocci, U. Bruzzo, D. Hernández Ruipérez and J. Muñoz Porras, Relatively stable bundles over elliptic fibrations, Math. Nachr. 238 (2002), 23-36. 


\section{DERIVED CATEGORIES OF IRREDUCIBLE PROJECTIVE CURVES}

BFM75 P. Baum, W. Fulton and R. MacPherson, Riemann-Roch for singular varieties, Publ. Math. Inst. Hautes Études Sci. 45 (1975), 101-145.

BBD82 A. Beilinson, J. Bernstein and P. Deligne, Faisceaux pervers, Astérisque 100 (1982).

BD03 L. Bodnarchuk and Yu. Drozd, Stable vector bundles over cuspidal cubics, Cent. Eur. J. Math. 1 (2003), 650-660.

BO97 A. Bondal and D. Orlov, Reconstruction of a variety from the derived category and groups of autoequivalences, Compositio Math. 125 (2001), 327-344.

Bri02 T. Bridgeland, Stability conditions on triangulated categories, math.AG/0212237.

BB05 I. I. Burban and I. M. Burban, Twist functors and D-branes, Ukraïn. Mat. Zh. 57 (2005), 18-31.

BD04b I. Burban and Yu. Drozd, Coherent sheaves on singular curves with simple double points and transversal intersections, Duke Math. J. 121 (2004), 189-229.

BD04a I. Burban and Yu. Drozd, Indecomposables of the derived categories of certain associative algebras, in Proc. ICRA-X conf., Fields Institute Communications, vol. 45 (American Mathematical Society, Providence, RI, 2004), 109-127, math.RT/0307062.

BK05 I. Burban and B. Kreußler, Fourier-Mukai transforms and semi-stable sheaves on nodal Weierstraß cubics, J. reine angew. Math. 584 (2005), 45-82.

BK04 I. Burban and B. Kreußler, On a relative Fourier-Mukai transform on genus one fibrations, Manuscripta Math., to appear.

Dou02 M. Douglas, Dirichlet branes, homological mirror symmetry, and stability, in Proc. Int. Congress of Mathematicians, Beijing, 2002, vol. III, Invited lectures (Higher Education Press, Beijing, 2002), 395-408.

Dro72 Yu. Drozd, Representations of commutative algebras, Funct. Anal. Appl. 6 (1972), 286-288; translation from Funkts. Anal. Prilozh. 6 (1972), 41-43.

DG01 Yu. Drozd and G.-M. Greuel, Tame and wild projective curves and classification of vector bundles, J. Algebra 246 (2001), 1-54.

FM03 R. Friedman and J. Morgan, Minuscule representations, invariant polynomials, and spectral covers, in Vector bundles and representation theory, eds S. D. Cutkosky et al., Columbia, MO, 2002, Contemporary Mathematics, vol. 322 (American Mathematical Society, Providence, RI, 2003), 1-41, math.AG/0011082.

Ful89 W. Fulton, Intersection theory, Ergebnisse der Mathematik und ihrer Grenzgebiete (3), vol. 2 (Springer, Berlin, 1989).

GKR04 A. Gorodentscev, S. Kuleshov and A. Rudakov, t-stabilities and t-structures on triangulated categories, Izv. Ross. Akad. Nauk Ser. Mat. 68 (2004), 117-150.

Gro77 A. Grothendieck (edited by I. Bucur), Groupes de classes des catégories abéliennes et triangulées. Complexes parfaits, in Semin. geom. algebr., Bois-Marie, 1965-66 (SGA 5), exposé VIII, Lecture Notes in Mathematics, vol. 589 (Springer, Berlin, 1977), 351-371.

HN75 G. Harder and M. S. Narasimhan, On the cohomology groups of moduli spaces of vector bundles on curves, Math. Ann. 212 (1975), 215-248.

LM93 H. Lenzing and H. Meltzer, Sheaves on a weighted projective line of genus one, and representations of a tubular algebra, in Representations of algebras, Ottawa, 1992, Canadian Mathematical Society Conf. Proc., vol. 14 (American Mathematical Society, Providence, RI, 1993), 313-337.

LM00 H. Lenzing and H. Meltzer, The automorphism group of the derived category for a weighted projective line, Comm. Algebra 28 (2000), 1685-1700.

Mel97 H. Meltzer, Tubular mutations, Colloq. Math. 74 (1997), 267-274.

Moz04 S. Mozgovoy, Classification of semi-stable sheaves on a rational curve with one node, Preprint (2004), math.AG/0410190.

Muk81 S. Mukai, Duality between $D(X)$ and $D(\hat{X})$ with its application to Picard sheaves, Nagoya Math. J. 81 (1981), 153-175.

Oda71 T. Oda, Vector bundles on an elliptic curve, Nagoya Math. J. 43 (1971), 41-72. 


\section{DERIVED CATEGORIES OF IRREDUCIBLE PROJECTIVE CURVES}

Orl97 D. Orlov, Equivalences of derived categories and K3 surfaces, J. Math. Sci. (New York) 84 (1997), 1361-1381.

PX97 L. Peng and J. Xiao, Root categories and simple Lie algebras, J. Algebra 198 (1997), $19-56$.

Pol02 A. Polishchuk, Classical Yang-Baxter equation and the $A_{\infty}$-constraint, Adv. Math. 168 (2002), 56-95.

Pol04 A. Polishchuk, Noncommutative two-tori with real multiplication as noncommutative projective varieties, J. Geom. Phys. 50 (2004), 162-187.

PS03 A. Polishchuk and A. Schwarz, Categories of holomorphic vector bundles on noncommutative two-tori, Commun. Math. Phys. 236 (2003), 135-159.

Rud97 A. Rudakov, Stability for an Abelian category, J. Algebra 197 (1997), 231-245.

ST01 P. Seidel and R. Thomas, Braid group actions on derived categories of coherent sheaves, Duke Math. J. 108 (2001), 37-108.

Tu93 L. Tu, Semistable bundles over an elliptic curve, Adv. Math. 98 (1993), 1-26.

Igor Burban burban@mathematik.uni-mainz.de

Johannes-Gutenberg Universität Mainz, Fachbereich Physik, Mathematik und Informatik, Institut für Mathematik, 55099 Mainz, Germany

Bernd Kreußler bernd.kreussler@mic.ul.ie

Mary Immaculate College, South Circular Road, Limerick, Ireland 